\title{
Analysis of the magnetic coupling in binuclear complexes. I. Physics of the coupling
}

\author{
Carmen J. Calzado \\ Laboratoire de Physique Quantique, IRSAMC, Université Paul Sabatier, 118, Route de Narbonne, \\ 31062 Toulouse, France \\ Jesús Cabrero \\ Departament de Química Física i Inorgànica and Institut d'Estudis Avançats, Universitat Rovira $i$ Virgili, \\ Pl. Imperial Tarraco, 1. 43005 Tarragona, Spain \\ Jean Paul Malrieu \\ Laboratoire de Physique Quantique, IRSAMC, Université Paul Sabatier, 118, Route de Narbonne, \\ 31062 Toulouse, France \\ Rosa Caballol \\ Departament de Química Física i Inorgànica and Institut d'Estudis Avançats, Universitat Rovira i Virgili, \\ Pl. Imperial Tarraco, 1. 43005 Tarragona, Spain
}

(Received 4 September 2001; accepted 6 November 2001)

\begin{abstract}
Accurate estimates of the magnetic coupling in binuclear complexes can be obtained from ab initio configuration interaction $(\mathrm{CI})$ calculations using the difference dedicated $\mathrm{CI}$ technique. The present paper shows that the same technique also provides a way to analyze the various physical contributions to the coupling and performs numerical analysis of their respective roles on four binuclear complexes of $\mathrm{Cu}\left(d^{9}\right)$ ions. The bare valence-only description (including direct and kinetic exchange) does not result in meaningful values. The spin-polarization phenomenon cannot be neglected, its sign and amplitude depend on the system. The two leading dynamical correlation effects have an antiferromagnetic character. The first one goes through the dynamical polarization of the environment in the ionic valence bond forms (i.e., the $\mathrm{M}^{+} \cdots \mathrm{M}^{-}$structures). The second one is due to the double excitations involving simultaneously single excitations between the bridging ligand and the magnetic orbitals and single excitations of the environment. This dispersive effect results in an increase of the effective hopping integral between the magnetic orbitals. Moreover, it is demonstrated to be responsible for the previously observed larger metal-ligand delocalization occurring in natural orbitals with respect to the Hartree-Fock ones. (c) 2002 American Institute of Physics. [DOI: 10.1063/1.1430740]
\end{abstract}

\section{INTRODUCTION}

The field of molecular and solid state magnetism arouses increasing interest in organic and organometallic chemistry as well as in material science. ${ }^{1-3}$ These systems are characterized by the existence of localized unpaired electrons, usually located on metallic ions. The properties of the material are governed by the interaction between their unpaired electrons on neighbor centers, which may be viewed as an effective interaction between site-centered spins, and mapped onto a Heisenberg-Dirac-Van Vleck Hamiltonian, ${ }^{4}$

$$
\hat{H}=-\sum_{i, j} J_{i j} \hat{S}_{i} \hat{S}_{j}
$$

For a bicentric system with $m_{s}= \pm 1 / 2$ on each center, the coupling constant $J={ }^{1} E-{ }^{3} E$ is negative for a singlet ground state (antiferromagnetism) and positive in the opposite case (ferromagnetism). $J$ is usually very small, ranging from a few hundreds to a few $\mathrm{cm}^{-1}$, and its calculation is a challenge for theoreticians. Quantum chemistry may be successfully applied for such evaluations. Numerous studies employ the density functional theory approach, mostly with the popular B3LYP exchange correlation potential, ${ }^{5-10}$ using
Noodleman's approximation, ${ }^{11}$ in which the magnetic coupling is evaluated from the difference between the Unrestricted (spin polarized) highest multiplet and the brokensymmetry (BS) determinant. Although this procedure may be useful, in particular to predict or to analyze trends in magneto-structural correlations, it suffers from two weaknesses: the dependence on the $V_{\mathrm{xc}}$ multiparametric exchange correlation potential and the spin contamination problems, especially severe for the broken-symmetry solution.

Properly using Noodleman's formula, i.e., taking into account the very small overlap between the magnetic orbitals in the BS solution, the B3LYP functional usually gives values of $J$ about twice the experimental ones. ${ }^{7,8,12}$ Moreover, this procedure does not allow to analyze the various contributions to $J$ and to check the validity of the semiempirical rationales historically proposed to interpret the sign and the magnitude of $J$ and its structural dependencies that will be briefly recalled.

$A b$ initio techniques that work with the exact Hamiltonian overcome these difficulties and limits provided that all the meaningful physical effects are incorporated in the treatment, resulting in a good agreement with experiment. 
Among the successful methods one may first quote the nonorthogonal configuration interaction (NOCI), ${ }^{13}$ which introduces a very limited number of valence bond configurations, including various ligand to metal charge transfer states. ${ }^{14,15}$ The list suffers some arbitrariness. The method requires specific optimization of the orbitals of each VB configuration and has not received numerous applications. More recently, the CASPT2 (Refs. 16 and 17) method has been used in this field. The second order expansion gives rather satisfactory results provided that the variational complete active space is large enough and properly chosen. ${ }^{18-21}$

In the recent past, an alternative configuration interaction (CI) technique has been designed for a direct evaluation of vertical energy differences, the difference dedicated configuration interaction (DDCI), ${ }^{22}$ which has been successfully employed in a wide series of studies, concerning molecular ${ }^{23-26}$ as well as solid state magnetic materials. ${ }^{27-30}$ An error smaller than $10 \mathrm{~cm}^{-1}$ is typical for a value of $J \sim 100$ $\mathrm{cm}^{-1}$. The definition of the DDCI space is based on second order quasidegenerate perturbation theory arguments as explained in our early papers in this domain. ${ }^{22,23}$ A secondorder perturbative approach has the advantage of providing a partition of the final $J$ value into a sum of different physical contributions $^{31}$ such as direct exchange, Anderson's antiferromagnetic (or kinetic exchange) contribution, spin polarization, etc. However the second order perturbative expansion is not numerically reliable because of convergence problems and arbitrariness in the choice of the zero order Hamiltonian. The DDCI method goes through an exact diagonalization and hence gains in accuracy, but the analysis of the various physical mechanisms involved in the coupling is not straightforward any more.

However, the usefulness of such a rigorous analysis is indubitable since it would allow to check the validity of the qualitative models, frequently used in an a posteriori rationalization and in the molecular design of new materials. ${ }^{1}$ The target of the present paper is to show that it is possible to combine numerical accuracy (going through large basis sets and long CI expansions) and analysis in terms of physical contributions. Four different antiferromagnetic systems have been considered in the present work, all of them involving two electrons on two $\mathrm{Cu}\left(d^{9}\right)$ sites: the $\left[\mathrm{Cu}_{2} \mathrm{Cl}_{6}\right]^{2-}$, the $\left[\mathrm{Cu}_{2}\left(\mu-\mathrm{N}_{3}\right)_{2}\left(\mathrm{NH}_{3}\right)_{6}\right]^{2+}$, and the $\mathrm{Cu}_{2}\left(\mu-\mathrm{CH}_{3} \mathrm{COO}\right)_{4}\left(\mathrm{H}_{2} \mathrm{O}\right)_{2}$ complexes and the $\mathrm{Cu}_{2} \mathrm{O}_{7}$ cluster, a model of the $\mathrm{La}_{2} \mathrm{CuO}_{4}$ perovskite. Section II describes the computational details. After recalling the valence only treatments in Sec. III, the theoretical development and the strategy of analysis are presented in Sec. IV and applied on these four binuclear systems. Finally, Sec. V reanalyzes the role of the different contributions when using natural orbitals instead of the triplet Hartree-Fock orbitals used in Secs. III and IV.

\section{DESCRIPTION OF THE MODELS AND COMPUTATIONAL DETAILS}

The four systems considered here, represented in Fig. 1, contain two $\mathrm{Cu}\left(d^{9}\right)$ centers, bridged by different ligands and with different relative positions. Figure 1(a) depicts our first model, the $\left[\mathrm{Cu}_{2} \mathrm{Cl}_{6}\right]^{2-}$, which magnetostructural depen- (a)
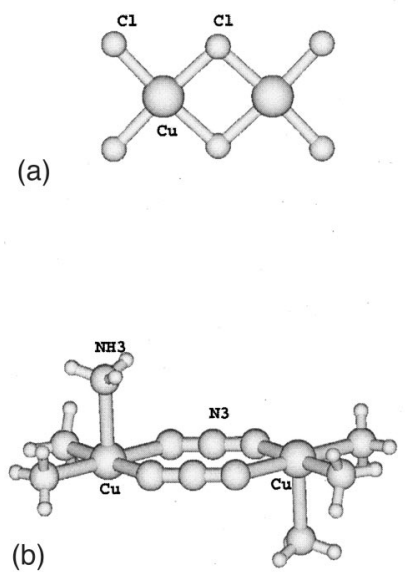

(c)

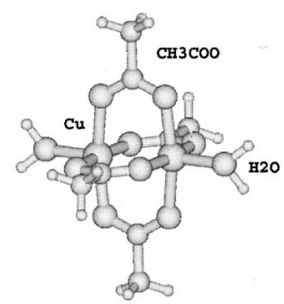

FIG. 1. Schematic representation of the four models considered: (a) the $\left[\mathrm{Cu}_{2} \mathrm{Cl}_{6}\right]^{2-}$ complex, in a planar geometry; (b) the $\left[\mathrm{Cu}_{2}\left(\mu-\mathrm{N}_{3}\right)_{2}\left(\mathrm{NH}_{3}\right)_{6}\right]^{2+}$ complex with end-to-end bridging azido ligands; (c) the $\mathrm{Cu}_{2}\left(\mu-\mathrm{CH}_{3} \mathrm{COO}\right)_{4}\left(\mathrm{H}_{2} \mathrm{O}\right)_{2}$ complex; (d) the $\mathrm{Cu}_{2} \mathrm{O}_{7}$ cluster.

dence has been extensively studied in the recent past. ${ }^{9,23,32,33}$ We have concentrated on the planar structure, where each $\mathrm{Cu}$ atom has a square planar coordination and bears its unpaired electron in a $d_{x y}$-type orbital [Fig. 2(a)]. The $\mathrm{Cu}-\mathrm{Cu}$ distance in the real complex is $3.44 \AA$. Experimentally, the planar structure of this complex belongs to the $C_{i}$ point group but only very slightly distorted from $D_{2 h}$, considered in the present work.

A similar situation is found in the $\left[\mathrm{Cu}_{2}\left(\mu-\mathrm{N}_{3}\right)_{2}\left(\mathrm{NH}_{3}\right)_{6}\right]^{2+}$ complex [Fig. 1(b)] with a square pyramidal coordination of the $\mathrm{Cu}$ centers [Fig. 2(b)]. The $\mathrm{Cu}-\mathrm{Cu}$ distance is $5.19 \AA$, larger than in the $\left[\mathrm{Cu}_{2} \mathrm{Cl}_{6}\right]^{2-}$ complex, but the magnetic coupling constant in the former is one order of magnitude larger than in the latter, showing that the end-to-end bridged $\left(\mathrm{N}_{3}\right)^{-}$groups play a crucial role in the exchange phenomenon. ${ }^{34,35}$ As in the precedent case, the experimental $C_{i}$ geometry has been symmetrized to $C_{2 h}$.

Regarding the $\mathrm{Cu}_{2}\left(\mu-\mathrm{CH}_{3} \mathrm{COO}\right)_{4}\left(\mathrm{H}_{2} \mathrm{O}\right)_{2}$ molecule [Fig. 1(c)], the $\mathrm{Cu}$ atoms have also a square pyramidal coordination, but are placed in parallel planes, bridged by four bidentate acetato-ligands and separated by $2.64 \AA$ only. Each $\mathrm{Cu}$ atom bears an unpaired electron in a $d_{x^{2}-y^{2}}$ orbital [Fig. 2(c)]. The experimental $C_{i}$ geometry ${ }^{36}$ has been used in the calculations. The nature of the magnetic interaction in this system was a matter of controversy during three decades: Do the two $d_{x^{2}-y^{2}}$ unpaired electrons interact directly via a $\delta$-bond or indirectly through the acetato-ligands? ${ }^{37}$ The experimental works of Güdel et al. ${ }^{38}$ and Ewald and Sinn ${ }^{39}$ together with the $a b$ initio calculation of de Loth et al. ${ }^{31}$ ruled out the $\delta$-bond hypothesis and showed the role of the acetato-ligand in the $\mathrm{Cu}-\mathrm{Cu}$ exchange.

The last considered system is a model of the antiferromagnetic perovskite $\mathrm{La}_{2} \mathrm{CuO}_{4}$. It consists of a $\mathrm{Cu}_{2} \mathrm{O}_{7}$ cluster [Fig. 1(d)], embedded in a set of point charges to mimic the Madelung field of the $\mathrm{La}_{2} \mathrm{CuO}_{4}$ lattice. The unpaired electron is placed in a $d_{x^{2}-y^{2}}$ orbital, but unlike the precedent models, the $\mathrm{Cu}$ atoms are bridged by just one oxygen ligand [Fig. 2(d)]. The magnetic coupling in this compound is quite large 


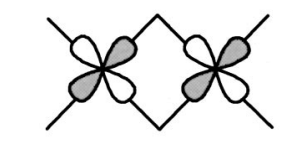

$\mathbf{a}$

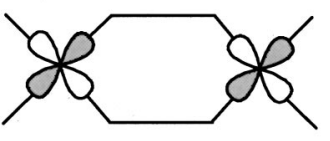

b
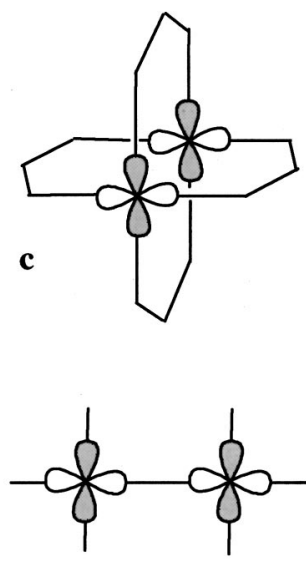

d
FIG. 2. Relative orientation of the magnetic orbitals in (a) the $\left[\mathrm{Cu}_{2} \mathrm{Cl}_{6}\right]^{2-}$ complex; (b) the $\left[\mathrm{Cu}_{2}\left(\mu-\mathrm{N}_{3}\right)_{2}\left(\mathrm{NH}_{3}\right)_{6}\right]^{2+}$ complex; (c) the $\mathrm{Cu}_{2}\left(\mu-\mathrm{CH}_{3} \mathrm{COO}\right)_{4}\left(\mathrm{H}_{2} \mathrm{O}\right)_{2}$ molecule; and (d) the $\mathrm{Cu}_{2} \mathrm{O}_{7}$ cluster.

(around $-1000 \mathrm{~cm}^{-1}$ ), well established from $\operatorname{Raman}^{40}$ and neutron diffraction ${ }^{41}$ experiments. Different theoretical approaches have been employed to evaluate $J$ [for instance, finite $^{8}$ and periodic ${ }^{42}$ DFT calculations, nonorthogonal CI (Ref. 14) and DDCI (Refs. 27, 28) calculations] the CI procedures giving the most correct results.

Since the four models presented here have been studied in previous independent works, different basis sets have been used in the $\mathrm{HF}-\mathrm{CI}$ calculations. In the $\left[\mathrm{Cu}_{2} \mathrm{Cl}_{6}\right]^{2-}$ complex $\mathrm{A}$, the azido-bridged complex $\mathrm{B}$, and copper acetate $\mathrm{C}$, an effective core potential of Barandiarán and Seijo has been employed for the $\mathrm{Cu}$ atoms, where the valence electrons are described by a $(9 s 6 p 6 d) /[3 s 3 p 4 d]$ basis set. ${ }^{43}$ ANO-type functions $^{44}$ have been used for $\mathrm{Cl}, \mathrm{N}$, and $\mathrm{H}$, with the following contractions: ANO-s.4s3p1d for $\mathrm{Cl}$, ANO- $s .3 s 2 p 1 d$ for the bridging $\mathrm{N}$ atoms, ANO- $s .3 s 2 p$ for the $\mathrm{N}$ atoms of the external $\mathrm{NH}_{3}$ groups, and ANO-s.2s for the $\mathrm{H}$ atoms. For the copper acetate $\mathrm{C}$, due to the size of the system, the effective core potentials proposed by Huzinaga ${ }^{45}$ have been used for the $\mathrm{C}$ and $\mathrm{O}$ atoms, with basis sets $(5 s 6 p 1 d) /[2 s 2 p 1 d]$ for oxygen atoms and $(5 s 5 p 1 d) /$ $[2 s 2 p 1 d]$ for the carbon atoms. In the $\mathrm{CH}_{3}$ groups and $\mathrm{H}_{2} \mathrm{O}$ molecules, minimal basis sets have been used, $(6 s 3 p) /[2 s 1 p]$ for $\mathrm{O}$ and $\mathrm{C}$, and $(3 s) /[1 s]$ for $\mathrm{H}^{46}$ Finally, in the $\mathrm{Cu}_{2} \mathrm{O}_{7}$ cluster $\mathrm{D}$, the Hay-Wadt pseudopotential and basis functions have been used for $\mathrm{Cu}$ atoms. ${ }^{47}$ For the oxygen atoms an all electron basis set $(10 s 5 p)$ contracted to $[3 s 2 p]$ has been employed, ${ }^{48}$ enlarged with a $d$ polarization function $(\alpha=0.85)$ in the bridging-oxygen atom.

The ROHF molecular orbitals have been obtained by using the MOLCAS 4.1 package. ${ }^{49}$ The CASDI (Ref. 50) program has been used in the CI calculations and the NATURAL (Ref. 51) program in the determination of the natural MOs.

\section{VALENCE-ONLY DESCRIPTION}

\section{A. Theory}

\section{One-band model}

From the early seventies, several qualitative models were proposed ${ }^{52-54}$ to interpret the physical factors govern- ing the magnetic coupling. Early analysis pointed out the existence of two antagonist contributions, $J=J_{F}+J_{\mathrm{AF}}$, where $F$ and AF indicate ferromagnetic and antiferromagnetic contributions, respectively. $J_{F}$ is attributed to the direct exchange between the magnetic orbitals (always ferromagnetic), and $J_{\mathrm{AF}}$ is interpreted through the delocalization effect that can only occur in the singlet state (hence antiferromagnetic) and is sometimes called kinetic exchange. These rationalizations may follow orthogonal valence bond (VB), ${ }^{52}$ nonorthogonal VB (Ref. 53) or valence configuration interaction (VCI) (Ref. 54) arguments, but in general they handle two electrons in two magnetic (local or symmetry-adapted) orbitals.

Let us consider the VCI and the orthogonal VB approaches for the simplest symmetrical $A^{\bullet}-B^{\bullet}$ system with two electrons in two orbitals. The orbitals are supposed to be previously determined, giving orthogonal core orbitals (closed shell) and magnetic orbitals. These magnetic orbitals may be either symmetry-adapted, $g$ and $u$, or their equivalent localized transforms, $a$ and $b$,

$$
g=\frac{a+b}{\sqrt{2}} \quad \text { and } \quad u=\frac{a-b}{\sqrt{2}}
$$

or

$$
a=\frac{g+u}{\sqrt{2}} \text { and } \quad b=\frac{g-u}{\sqrt{2}} .
$$

With two active electrons in the magnetic orbitals, the CI space is limited to four determinants. In the orthogonal VB approach, for the $M_{s}=0$ component, there are two neutral determinants,

$$
|a \bar{b}\rangle=\mid \text { core } a \bar{b}\rangle \quad \text { and } \quad|b \bar{a}\rangle=\mid \text { core } b \bar{a}\rangle,
$$

and two ionic determinants,

$$
|a \bar{a}\rangle=\mid \text { core } a \bar{a}\rangle \text { and }|b \bar{b}\rangle=\mid \text { core } b \bar{b}\rangle .
$$

The CI matrix takes the form

$$
\begin{gathered}
|a \bar{b}\rangle \\
|b \bar{a}\rangle \\
|a \bar{a}\rangle \\
|b \bar{b}\rangle
\end{gathered}\left[\begin{array}{cccc}
0 & K_{a b} & t_{a b} & t_{a b} \\
K_{a b} & 0 & t_{a b} & t_{a b} \\
t_{a b} & t_{a b} & U & K_{a b} \\
t_{a b} & t_{a b} & K_{a b} & U
\end{array}\right],
$$

where the energy of the neutral VB determinants is considered as the energy origin. The direct exchange, $K_{a b}$ $=\left\langle a \bar{b}\left|1 / r_{12}\right| b \bar{a}\right\rangle$, is necessarily positive since it is the selfrepulsion of the $a b$ overlap electronic distribution, as well as $U$, the energy difference between the ionic and the neutral forms. The hopping integral $t_{a b}=\langle a \bar{b}|\hat{H}| a \bar{a}\rangle=\langle a|\hat{F}| b\rangle$, where $\hat{F}$ is the Fock operator, is the coupling between the neutral and the ionic VB forms. As shown by Hay et al. ${ }^{54}$ this integral can also be expressed by the diagonal Fock elements of the symmetry adapted orbitals, $t_{a b}=\frac{1}{2}\left(F_{g g}\right.$ $\left.-F_{u u}\right)$.

The four solutions are:

(i) $\quad$ in the $u$ symmetry: 
(a) a purely neutral triplet state,

$\left|T_{u}\right\rangle=\frac{1}{\sqrt{2}}(|a \bar{b}\rangle-|b \bar{a}\rangle)$

of energy

${ }^{3} E_{u}=-K_{a b}$.

We also refer to this $M_{s}=0$ component of the triplet configuration as

$T_{a b}^{0}=\frac{1}{\sqrt{2}}(a \bar{b}-b \bar{a}) ;$

(b) a purely ionic singlet state,

$\left|S_{u}\right\rangle=\frac{1}{\sqrt{2}}(|a \bar{a}\rangle-|b \bar{b}\rangle)$

of energy

${ }^{1} E_{u}=U-K_{a b}$;

(ii) in the $g$ symmetry, two singlet states which are mixtures of the neutral and the ionic components:

(a) the lowest one is essentially neutral,

$\left|S_{g}\right\rangle=\delta(|a \bar{b}\rangle+|b \bar{a}\rangle)+\gamma(|a \bar{a}\rangle+|b \bar{b}\rangle), \quad(\delta>\gamma>0)$.

Its energy is

${ }^{1} E_{g}=K_{a b}+\frac{U-\sqrt{U^{2}+16 t_{a b}^{2}}}{2}$.

It is also useful to define the singlet configuration,

$S_{a b}=\frac{1}{\sqrt{2}}(a \bar{b}+b \bar{a})$;

(b) the second one is essentially ionic,

$\left|S_{g}^{\prime}\right\rangle=-\gamma(|a \bar{b}\rangle+|b \bar{a}\rangle)+\delta(|a \bar{a}\rangle+|b \bar{b}\rangle)$,

and much higher in energy,

${ }^{1} E_{g}^{\prime}=K_{a b}+\frac{U+\sqrt{U^{2}+16 t_{a b}^{2}}}{2}$.

The singlet-triplet energy separation is given by

$$
J=\Delta E_{S T}={ }^{1} E_{g}-{ }^{3} E_{u}=2 K_{a b}+\frac{U-\sqrt{U^{2}+16 t_{a b}^{2}}}{2} .
$$

When $U \gg\left|t_{a b}\right|$, a power expansion, equivalent to a perturbation of the neutral singlet by the ionic one, is possible and the coupling constant tends to ${ }^{52}$

$$
J=2 K_{a b}-\frac{4 t_{a b}^{2}}{U}
$$

where the opposite signs of both contributions become evident.

It may be worth to introducing a diagrammatic picture of the coupling. The two neutral VB determinants may be depicted as in Diagram 1:
Diagram 1
$|a \bar{b}\rangle=\quad \frac{a}{\ll}$
$|b \bar{a}\rangle=\stackrel{\longleftrightarrow}{\ll}$

where the double arrows indicate the magnetic orbitals. Hereafter, simple left-to-right arrows $\rightarrow$ symbolize holes, i.e., inactive doubly occupied orbitals, from which an electron is excited, and simple right-to-left arrows $\leftarrow$ symbolize particles, i.e., virtual orbitals, into which electrons are excited.

The direct exchange appears as a first order interaction, represented in Diagram 2:

Diagram 2

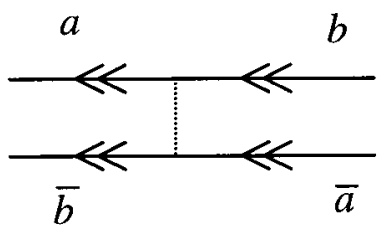

$$
|a \bar{b}\rangle a \uparrow \downarrow b \quad a \uparrow \uparrow+\downarrow|b \bar{a}\rangle
$$

while the kinetic exchange is a second order effect, as shown in Diagram 3:

Diagram 3
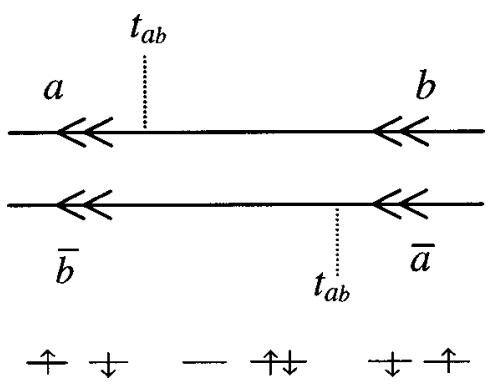

The same result is obtained when working with symmetryadapted MOs,

$$
\begin{aligned}
& \left|S_{g}\right\rangle=\lambda|g \bar{g}\rangle-\mu|u \bar{u}\rangle, \quad\left|T_{u}\right\rangle=\frac{1}{\sqrt{2}}(|g \bar{u}\rangle-|u \bar{g}\rangle), \\
& \left|S_{g}^{\prime}\right\rangle=\mu|g \bar{g}\rangle+\lambda|u \bar{u}\rangle, \quad\left|S_{u}\right\rangle=\frac{1}{\sqrt{2}}(|g \bar{u}\rangle+|u \bar{g}\rangle) .
\end{aligned}
$$

The coefficients in both approaches are related by

$$
2 \delta=\lambda+\mu \quad \text { and } \quad 2 \gamma=\lambda-\mu .
$$

When $|t / U|$ is small, $\lambda$ and $\mu$ are both close to $1 / \sqrt{2}$ and $\gamma$ is small.

The relations that give the values of $K_{a b}, t_{a b}$, and $U$ from the solutions of the $\left(2 e^{-} / 2 \mathrm{MO}\right) \mathrm{CI}$ are easily established,

$$
K_{a b}=\frac{{ }^{1} E_{g}+{ }^{1} E_{g}^{\prime}-{ }^{3} E_{u}-{ }^{1} E_{u}}{4},
$$




$$
\begin{aligned}
& U={ }^{1} E_{u}-{ }^{3} E_{u}, \\
& t_{a b}=-\frac{\sqrt{\left({ }^{1} E_{g}^{\prime}-{ }^{1} E_{g}\right)^{2}-\left({ }^{1} E_{u}-{ }^{3} E_{u}\right)^{2}}}{4} .
\end{aligned}
$$

Hence this variational CI calculation enables us to evaluate the direct exchange, $K_{a b}$, as well as the hopping integral, $t_{a b}$, and the on-site repulsion, $U$. Analogously, effective $K_{a b}, t_{a b}$, and $U$ values that include the effects of the dynamic correlation can be extracted in an exact way from large CI expansions, as will be shown in part II of this work. $^{58}$

\section{Two-band model}

Analogous elementary pictures may explicitly introduce ligand orbitals, when the exchange between the metal centers is supposed to proceed through a bridging-ligand in a $A^{\bullet}-L-B^{\bullet}$ structure. For simplicity, the ligand orbitals will be limited to a unique doubly occupied orbital, $l$. The two neutral VB determinants may be depicted as in Scheme 1:

Scheme 1

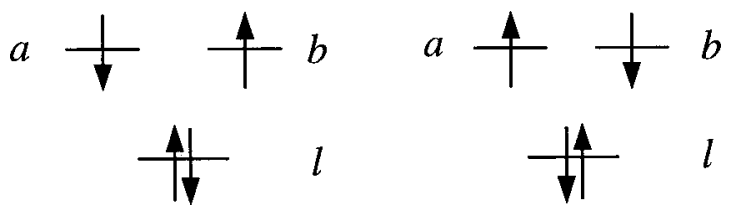

In addition to the previously discussed direct coupling, a fourth-order indirect coupling between them is possible that proceeds through a ligand to metal charge transfer (LMCT) process, according to Scheme 2:

Scheme 2

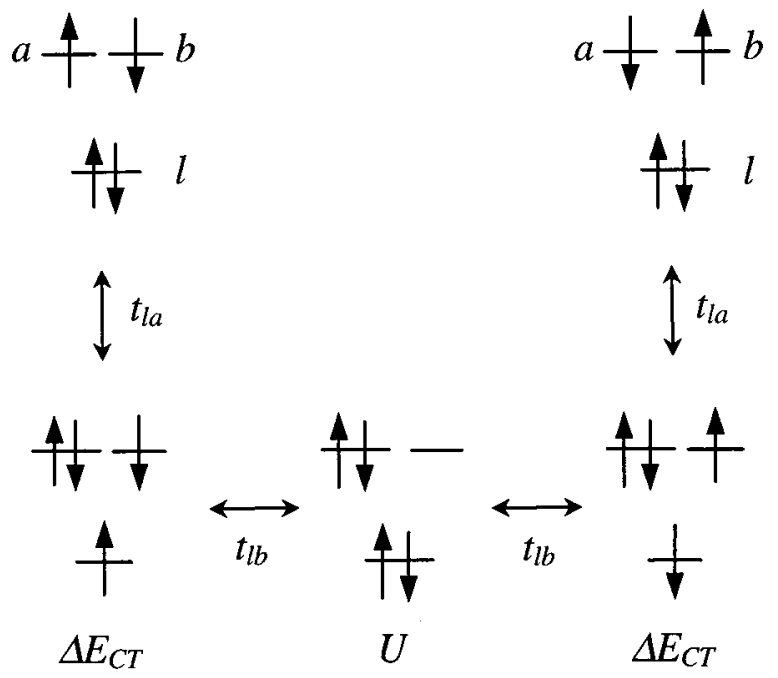

where $\Delta E_{\mathrm{CT}}$ is the excitation energy (hence, positive) to the ligand to metal charge transfer states $\left(A^{-} L^{+} B^{\bullet}\right)$. Obviously, there is an equivalent contribution involving $\left(A^{\bullet} L^{+} B^{-}\right)$intermediate. The diagrammatic representation of one of them is shown in Diagram 4:
Diagram 4

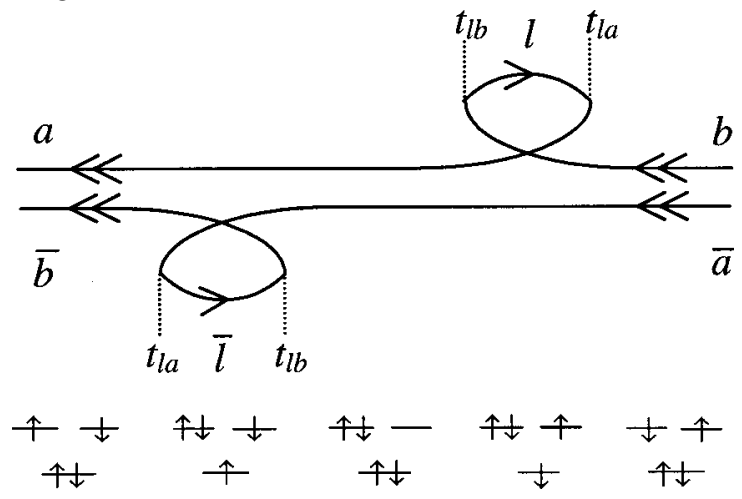

The corresponding contribution to $J$ is antiferromagnetic,

$$
J \longleftarrow-\frac{4 t_{l a}^{2} t_{l b}^{2}}{\Delta E_{\mathrm{CT}}^{2} U}
$$

and is the usual interpretation of superexchange. The contribution of the consecutive charge transfer processes through the ligand may be included in an effective hopping integral,

$$
t_{a b}^{\mathrm{eff}}=t_{a b}+\frac{t_{l a} t_{l b}}{\Delta E_{\mathrm{CT}}}
$$

and when $t_{a b}$ is small,

$$
J=2 K_{a b}-\frac{4\left(t_{a b}^{\mathrm{eff}}\right)^{2}}{U},
$$

which returns to the one-band model, Eq. (17). In principle, other mechanisms proceeding through the double charge transfer $\left(A^{-} L^{++} B^{-}\right)$intermediates (of relative energy $\left.\Delta E_{2 \mathrm{CT}}\right)$ may also be considered giving a contribution,

$$
J \leftarrow-\frac{8 t_{l a}^{2} t_{l b}^{2}}{\Delta E_{\mathrm{CT}}^{2} \Delta E_{2 \mathrm{CT}}} .
$$

A typical representation is Diagram 5:

Diagram 5

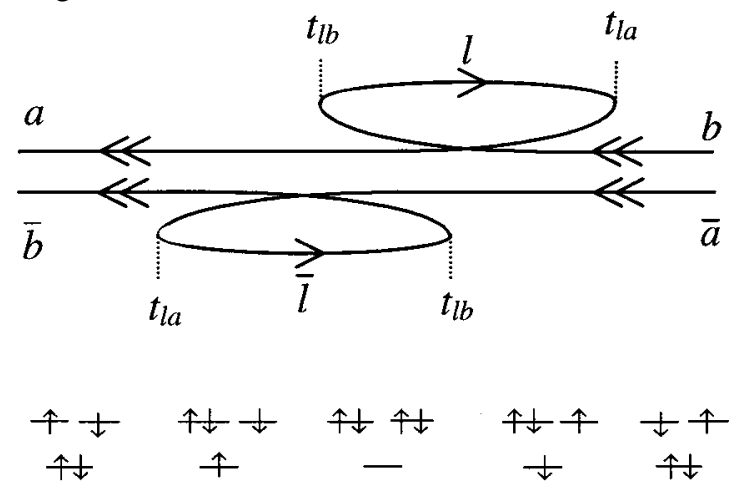

This double ligand to metal transfer is known in solid state physics as the Goodenough mechanism. ${ }^{55,56}$ Since the relative energy of the doubly ionic structure $\Delta E_{2 \mathrm{CT}}$ is certainly larger than $U$ this mechanism may be supposed to bring very small contributions, although it has been invoked sometimes. $^{29,56}$

\section{B. Numerical results}

The present section reports calculations of the valenceonly CASCI ( $2 e^{-}$in $\left.2 \mathrm{MOs}\right)$ for the four model problems. It 
TABLE I. Valence-only description with ROHF molecular orbitals. Direct exchange, $K_{a b}$, hopping integral, $t_{a b}$, on-site repulsion, $U$, coupling constant, $J$. All results in $\mathrm{cm}^{-1}$.

\begin{tabular}{lcccc}
\hline \hline & {$\left[\mathrm{Cu}_{2} \mathrm{Cl}_{6}\right]^{2-}$} & {$\left[\mathrm{Cu}_{2}\left(\mathrm{~N}_{3}\right)_{2}\left(\mathrm{NH}_{3}\right)_{6}\right]^{2+}$} & $\mathrm{Cu}_{2}\left(\mathrm{CH}_{3} \mathrm{COO}\right)_{4}\left(\mathrm{H}_{2} \mathrm{O}\right)_{2}$ & $\mathrm{Cu}_{2} \mathrm{O}_{7}$ \\
\hline$d_{\mathrm{Cu}-\mathrm{Cu}}(\AA)$ & 3.44 & 5.19 & 2.64 & 3.78 \\
$2 K_{a b}$ & 27 & 12 & 4 & 67 \\
$2 t_{a b}$ & -1756 & -4436 & -2085 & -7917 \\
$U\left(\times 10^{-4}\right)$ & 19.0 & 20.8 & -19.1 & 19.4 \\
$J$ & +11 & -82 & $-286^{\mathrm{c}},-294 \pm 4^{\mathrm{d}}$ & $-1032 \pm 48^{\mathrm{e}}$ \\
$J_{\exp }$ & $0,-40^{\mathrm{a}}$ & $<-800^{\mathrm{b}}$ & & $-1081 \pm 40^{\mathrm{f}}$ \\
\hline \hline
\end{tabular}

${ }^{\mathrm{a}}$ Reference 62.

${ }^{\mathrm{b}}$ Reference 63.

${ }^{\mathrm{c}}$ Reference 64.
${ }^{\mathrm{d}}$ Reference 38 .

${ }^{\mathrm{e}}$ Reference 40.

${ }^{\mathrm{f}}$ Reference 41. has been shown ${ }^{57}$ that the orbitals of the ROHF triplet and of the $(2 e / 2 \mathrm{MO}) \mathrm{CASSCF}$ singlet are almost identical. The results presented in Table I are obtained from the triplet state MOs and show that:

(1) The $K_{a b}$ ferromagnetic direct exchange is small, without a clear correlation with the $\mathrm{Cu}-\mathrm{Cu}$ distance, since the orientation of the magnetic orbitals (Fig. 2) is not the same and the tails on the bridging-ligands depend on their chemical nature.

(2) The $t_{a b}$ hopping integral contribution is quite different from one system to another. Comparing the chloride and the azido complexes, both with $d_{x y}$ magnetic orbitals (Fig. 2), makes evident the role of the delocalization tails on the bridging ligand since $t_{a b}$ is larger in the second one despite the larger $\mathrm{Cu}-\mathrm{Cu}$ distance. The energy difference between the neutral and the ionic VB components, $U$, is very large (around $2 \times 10^{5} \mathrm{~cm}^{-1}, \sim 24-25$ $\mathrm{eV}$ ), much larger than usually assumed in model Hamiltonians. One may notice that this quantity is almost invariant of the ligand and of the metal-metal distance. This near-invariance is in better agreement with the onsite repulsion of the simple Hubbard model, ${ }^{52}$ than with more elaborated methods ${ }^{54}$ in which

$U=J_{a a}-J_{a b}$,

$J_{a a}$ and $J_{a b}$ being the one-center and two-centers Coulomb repulsions, respectively.

(3) The overall $J$ value is very far from the experimental one. It is even of the wrong sign for the chloride complex. It shows that a lot of physics is lacking at this stage which nevertheless contains the two leading factors usually involved for interpretation. It is clear that the antiferromagnetic contribution is grossly underestimated.

\section{BEYOND THE VALENCE-ONLY DESCRIPTION: DYNAMICAL CORRELATION EFFECTS}

\section{A. The Brillouin's theorem and its consequences}

At this stage it is important to discuss the effects of the orbitals used in the calculations. In Secs. III and IV, selfconsistent orbitals obtained from a variational restricted open-shell Hartree-Fock calculation of the triplet state are used. These orbitals satisfy the Brillouin's theorem, which consequences can be summarized as follows. When acting on the $T_{u}$ triplet state, the single excitations $a_{i}^{+} a_{j}$ (where $a$ and $a^{+}$are annhilation and creation operators, respectively) lead to $a_{i}^{+} a_{j} T_{u}$ determinants that do not interact with $T_{u}$,

$$
\left\langle a_{i}^{+} a_{j} T_{u}|\hat{H}| T_{u}\right\rangle=0 .
$$

Three types of single excitations are to be distinguished:

(1) The $1 \mathrm{~h}$ determinants correspond to the single excitations that send one electron from an occupied $h$ orbital to the $a$ magnetic orbital, $a_{a}^{+} a_{h}$ or $a_{\bar{a}}^{+} a_{\bar{h}}^{-},|h \bar{h}(a \bar{b}-b \bar{a})\rangle$ $\Rightarrow|h \bar{a} a \bar{b}-a \bar{h} b \bar{a}\rangle$. The Brillouin's theorem imposes $\left\langle a\left|\hat{h}_{c}+\hat{J}_{a}+\hat{J}_{b}\right| h\right\rangle=0$,

where $\hat{h}_{c}$ represents the Fock operator for the closed shells of the system,

$\hat{h}_{c}=\hat{h}+\sum_{k \in \text { core }} 2 \hat{J}_{k}-\hat{K}_{k}$,

$\hat{J}$ and $\hat{K}$ being the usual Coulomb and exchange operators.

(2) The $1 p$ determinant set contains all the single excitations from the magnetic orbitals to the virtual set, $a_{p}^{+} a_{a}$ or $a_{\bar{p}}^{+} a_{\bar{a}},|h \bar{h}(a \bar{b}-b \bar{a})\rangle \Rightarrow|h \bar{h} p \bar{b}-h \bar{h} b \bar{p}\rangle$. The Brillouin's theorem imposes in this case,

$\left\langle p\left|\hat{h}_{c}+\hat{J}_{a}-\hat{K}_{a}+\hat{J}_{b}-\hat{K}_{b}\right| a\right\rangle=0$.

(3) For the $1 h+1 p$ excitations, $a_{p}^{+} a_{h}$, the Brillouin's theorem gives

$\left\langle p\left|\hat{h}_{c}+\hat{J}_{a}-\frac{\hat{K}_{a}}{2}+\hat{J}_{b}-\frac{\hat{K}_{b}}{2}\right| h\right\rangle=0$.

This condition implies that the state obtained under the action of the singlet $a_{p}^{+} a_{h}+a_{\bar{p}}^{+} a_{\bar{h}}^{-}$excitations, $S_{h p}=1 / \sqrt{2}(h \bar{p}$ $+p \bar{h})$ on the triplet state,

$$
\left|S_{h p} \cdot T_{u}\right\rangle=\left|S_{h p} \cdot T_{a b}^{0}\right\rangle=\frac{1}{2}|(h \bar{p}+p \bar{h})(a \bar{b}-b \bar{a})\rangle
$$

does not interact with it,

$$
\left\langle S_{h p} \cdot T_{u}|\hat{H}| T_{u}\right\rangle=0 .
$$


It is easy to show that the same excitation acting on the singlet state,

$$
S_{h p} \cdot S_{a b}=\frac{1}{2}|(h \bar{p}+p \bar{h})(a \bar{b}+b \bar{a})\rangle,
$$

satisfies a similar equation,

$$
\left\langle S_{h p} \cdot S_{a b}|\hat{H}| S_{a b}\right\rangle=0,
$$

where $S_{a b}=1 / \sqrt{2}(a \bar{b}+b \bar{a})$ was defined in Eq. (13).

In summary, single excitations from the core to the virtual space do not interact with any of both states. Single excitations from the core to the active orbitals, Eq. (29), or from the active orbitals to the virtual ones, Eq. (31), do not interact with the SCF triplet state but they can give small interactions with the singlet that have to be carefully analyzed. As a consequence, the ligand-to-metal charge transfer excitations responsible for the delocalization tails on the ligand do not interact with the triplet state. It is shown in Sec. IV B 1 a that their interaction with the singlet is also extremely small. The off-diagonal elements $t_{l a}$ and $t_{l b}$ of the Fock operator are null and consequently the superexchange mechanisms, Eq. (23) and Eq. (26), do not contribute. The variational optimization of the orbitals in the SCF procedure defines an optimal delocalization between the ligand and the magnetic centers in such a way that these effects are incorporated in the valence-only CI.

\section{B. Taking only the neutral determinants as model space}

\section{Theory}

Following the logics of a previous work, ${ }^{31}$ the quasidegenerated perturbation theory may be used as a guideline to understand the effective coupling between the neutral forms, $|a \bar{b}\rangle$ and $|b \bar{a}\rangle$. The perturbative analysis is essentially conceptual, since the numerical analysis presented here is based on variational calculations. These two determinants may be considered as defining a physically meaningful twodimensional model space, upon which quasidegenerate perturbation theory allows to build an effective Hamiltonian, $\hat{H}_{\text {eff }}$. Its off-diagonal matrix element gives $J$ $=2\left\langle a \bar{b}\left|\hat{H}_{\mathrm{eff}}\right| b \bar{a}\right\rangle$. Up to the second order,

$$
\left\langle a \bar{b}\left|\hat{H}_{\mathrm{eff}}^{(2)}\right| b \bar{a}\right\rangle=K_{a b}+\sum_{|\alpha\rangle \neq|a \bar{b}\rangle,|b \bar{a}\rangle} \frac{\langle a \bar{b}|\hat{H}| \alpha\rangle\langle\alpha|\hat{H}| b \bar{a}\rangle}{E_{0}^{(0)}-E_{\alpha}^{(0)}},
$$

where $E_{0}^{(0)}=E_{|a \bar{b}\rangle}$. The second order contributions may be schematized as in Diagram 6:

Diagram 6

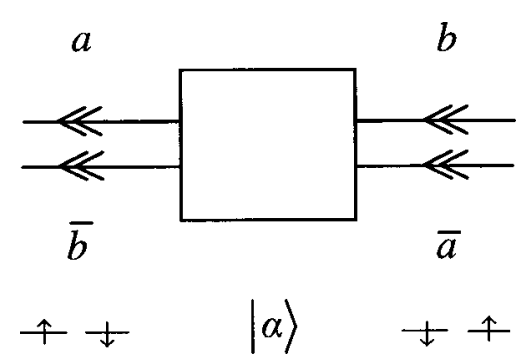

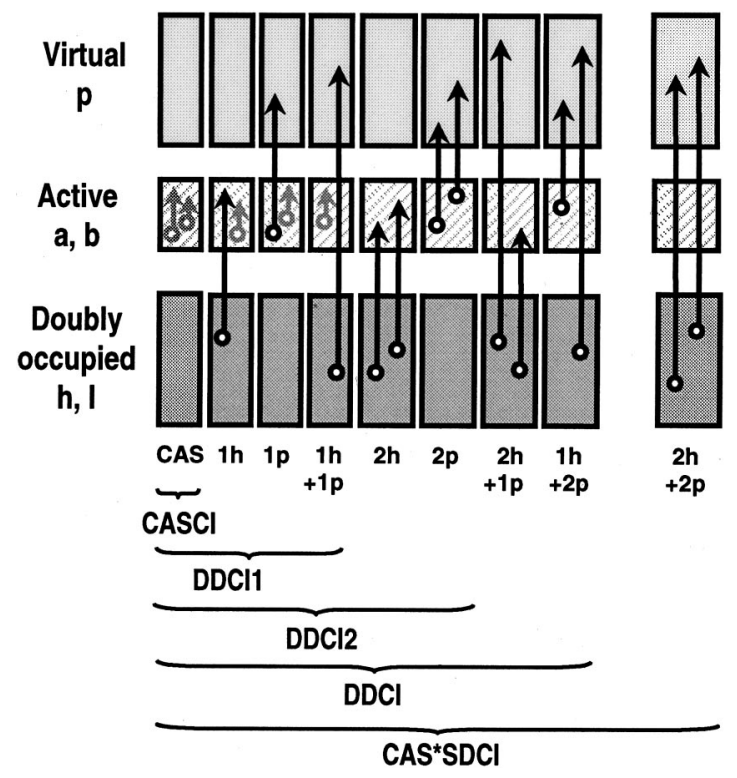

FIG. 3. Schematic representation of the various excitation operators leading to the relevant CI spaces.

In the preceding section, the role of $|\alpha\rangle=|a \bar{a}\rangle,|b \bar{b}\rangle$ has been analyzed. Other outer-space determinants can be obtained by excitations involving nonvalence (inactive) orbitals, either core-orbitals or virtual orbitals. The list of determinants contributing up to the second order as well as the corresponding physical contributions have been discussed by de Loth et al. ${ }^{31}$

The determinants of this list will be labeled hereafter by the number $n$ of the excited electrons from the core or holes, $n h$, and the number $m$ of promoted electrons to the virtual space or particles, $m p$. Up to the second order, $|\alpha\rangle$ involve five types of determinants: $1 h, 1 p, 1 h+1 p, 2 h$, and $2 p$, as represented in Fig. 3.

a. The $1 \mathrm{~h}$ and $1 p$ determinants. Among the previously defined $1 h$ determinants, the most important are the ligand to metal $(L \rightarrow M)$ charge transfer (LMCT) states, which do not interact with the triplet due to Brillouin's theorem, but lead to a small interaction with the singlet state,

$$
\begin{gathered}
\left\langle\frac{1}{\sqrt{2}}(h \bar{b}+b \bar{h}) a \bar{a}|\hat{H}| \frac{1}{\sqrt{2}} h \bar{h}(a \bar{b}+b \bar{a})\right\rangle \\
=2\left\langle h\left|\hat{K}_{b}\right| a\right\rangle=2(h b, a b),
\end{gathered}
$$

where the conventional notation of bielectronic integrals is used. The integral,

$$
\left\langle i(1) j(2)\left|\frac{1}{r_{l 2}}\right| k(1) l(2)\right\rangle=\langle i j \mid k l\rangle=(i k, j l)
$$

represents the interaction between the $i k$ and $j l$ overlap distributions. Since the $A$ and $B$ magnetic centers are usually well separated, the $a b$ distribution is very small. This results in a small integral and consequently small coefficients of the LMCT states in the singlet wave function, typically of the order of $10^{-2}$. 
Similarly, the $1 p$ determinants, which involve metal to ligand charge transfer excitations, do not interact with the triplet state, but their interaction with the singlet state is

$$
\begin{aligned}
& \left\langle\frac{1}{\sqrt{2}} h \bar{h}(a \bar{p}+p \bar{a})|\hat{H}| \frac{1}{\sqrt{2}} h \bar{h}(a \bar{b}+b \bar{a})\right\rangle \\
& =2\left\langle p\left|\hat{K}_{a}\right| b\right\rangle=2(p a, a b) .
\end{aligned}
$$

As in the precedent case, for two distant magnetic centers the magnitude of this integral is small and therefore the coefficients of the $1 p$ determinants on the singlet wave function are small as well.

$b$. The $1 h+1 p$ determinants. The $1 h+1 p$ determinants belong to three different classes.

(i) The pure singlet excitations in the inactive part, $S_{h p}$, represented in Scheme 3:

Scheme 3

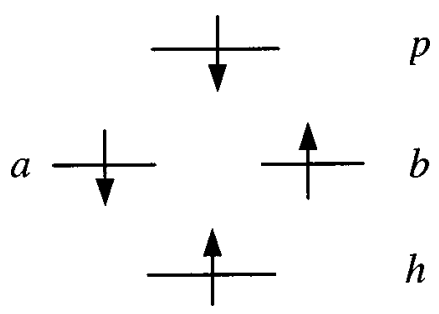

act on the $S_{a b}$ and $T_{a b}^{0}$ model space states giving the singlet, $S_{h p} \cdot S_{a b}=\frac{1}{2}|(h \bar{p}+p \bar{h})(a \bar{b}+b \bar{a})\rangle$, and the triplet, $S_{h p} \cdot T_{a b}^{0}$ $=\frac{1}{2}|(h \bar{p}+p \bar{h})(a \bar{b}-b \bar{a})\rangle$, defined in Eqs. (35) and (33), respectively.

The interaction of these single-excited states with the singlet and triplet states does not bring any contribution to the $S-T$ energy difference, due to the Brillouin's theorem, as shown in Sec. IV A.

(ii) The triplet excitations in the inactive part, as shown in Scheme 4:

Scheme 4

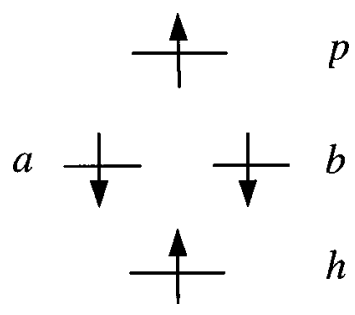

introduce the spin polarization effect. The inactive part becomes

$$
T_{h p}^{0}=\frac{1}{\sqrt{2}}(h \bar{p}-p \bar{h}), \quad T_{h p}^{+}=h p, \quad T_{h p}^{-}=\bar{h} \bar{p},
$$

which acting on the model space gives

(1) two triplet states:

$$
\begin{aligned}
& T_{h p}^{0} \cdot S_{a b}=\frac{1}{2}|(h \bar{p}-p \bar{h})(a \bar{b}+b \bar{a})\rangle, \\
& \frac{1}{\sqrt{2}}\left(T_{h p}^{+} \cdot T_{a b}^{-}-T_{h p}^{-} \cdot T_{a b}^{+}\right)=\frac{1}{\sqrt{2}}|(h p \bar{a} \bar{b}-\bar{h} \bar{p} a b)\rangle ;
\end{aligned}
$$

one singlet state:

$$
\frac{1}{\sqrt{3}}\left(T_{h p}^{+} \cdot T_{a b}^{-}+T_{h p}^{-} \cdot T_{a b}^{+}-T_{h p}^{0} \cdot T_{a b}^{0}\right) .
$$

The interaction of the triplet states with $T_{a b}^{0}$ brings the following contributions:

$$
\begin{aligned}
& \left\langle T_{h p}^{0} \cdot S_{a b}|\hat{H}| T_{a b}^{0}\right\rangle=\frac{1}{\sqrt{2}}\left\langle p\left|\hat{K}_{a}-\hat{K}_{b}\right| h\right\rangle, \\
& \frac{1}{\sqrt{2}}\left\langle T_{h p}^{+} \cdot T_{a b}^{-}-T_{h p}^{-} \cdot T_{a b}^{+}|\hat{H}| T_{a b}^{0}\right\rangle=\left\langle p\left|\hat{K}_{a}+\hat{K}_{b}\right| h\right\rangle .
\end{aligned}
$$

For the singlet state,

$$
\begin{aligned}
\frac{1}{\sqrt{3}}\left\langle T_{h p}^{+} \cdot T_{a b}^{-}+T_{h p}^{-} \cdot T_{a b}^{+}-T_{h p}^{0} \cdot T_{a b}^{0}|\hat{H}| S_{a b}\right\rangle \\
=-\frac{3}{\sqrt{2}}\left\langle p\left|\hat{K}_{a}-\hat{K}_{b}\right| h\right\rangle
\end{aligned}
$$

The local $\hat{K}_{a}$ and $\hat{K}_{b}$ exchange operators introduce spin polarization of the inactive orbitals (or closed shells). The total spin polarization energy in the triplet state is

$$
{ }^{3} E_{h p}^{(2)}=-\frac{1 / 2\left\langle p\left|\hat{K}_{a}-\hat{K}_{b}\right| h\right\rangle^{2}+\left\langle p\left|\hat{K}_{a}-\hat{K}_{b}\right| h\right\rangle^{2}}{\Delta E_{h \rightarrow p}},
$$

where $\Delta E_{h \rightarrow p}$ is a positive quantity representing the excitation energy of the promoted inactive electrons, $E_{|h \bar{p} a \bar{b}\rangle}$ $-E_{|h \bar{h} a \bar{b}\rangle}$.

The total spin polarization energy of the singlet state is

$$
{ }^{1} E_{h p}^{(2)}=-\frac{3 / 2\left\langle p\left|\hat{K}_{a}-\hat{K}_{b}\right| h\right\rangle^{2}}{\Delta E_{h \rightarrow p}} .
$$

The contributions to both states are significant. The terms $\left\langle p\left|\hat{K}_{a}\right| h\right\rangle^{2} / \Delta E_{h \rightarrow p}$ (resp. $b$ ), which introduce the spin polarization of the electrons around each magnetic center, contribute equally to the singlet and the triplet states and cancel in the transition. Hence, the contribution to the $S-T$ energy splitting comes only from the crossed terms,

$$
J \leftarrow+\frac{4\left\langle h\left|\hat{K}_{a}\right| p\right\rangle\left\langle p\left|\hat{K}_{b}\right| h\right\rangle}{\Delta E_{h \rightarrow p}} .
$$

This contribution, labeled $\varepsilon_{D S P}^{2}$ in de Loth et al., ${ }^{31}$ is nonnegligible only when the $h p$ distribution is important near $A$ and $B$, i.e., for $h$ and $p$ being bridging-ligand orbitals. Its sign cannot be predicted since it depends on the signs of the $h p$ distribution near the magnetic centers, which depends on the ligand. The corresponding second-order diagrams to the coupling are of Diagram 7 type. The contribution of the spin polarization to $J$ is easily identified by extending the CI space, spanned by the valence CAS, with the determinants of the $T_{h p}^{+} \cdot T_{a b}^{-}$and $T_{h p}^{-} \cdot T_{a b}^{+}$type, which are responsible for the differential effect, 
Diagram 7
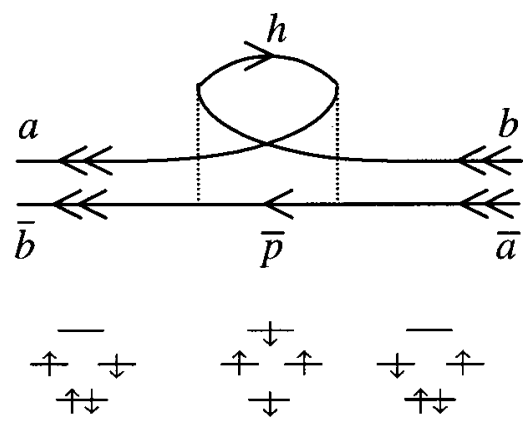

$+$
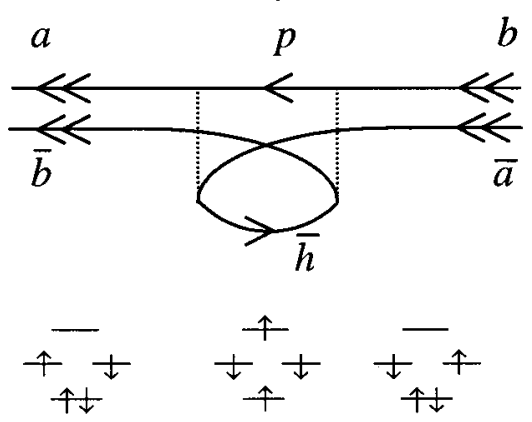

(iii) The inactive single excitations with a spatial change in the active part, shown in Scheme 5, namely, the single excitations on the ionic VB forms (singlet by nature) give $S_{h p} \cdot a \bar{a}$ and $S_{h p} \cdot b \bar{b}$ singlets and $T_{h p}^{0} \cdot a \bar{a}$ and $T_{h p}^{0} \cdot b \bar{b}$ triplets:

Scheme 5

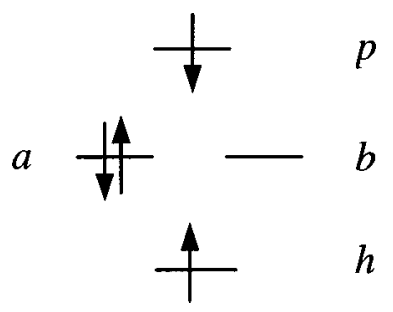

The interaction of $S_{h p} \cdot a \bar{a}$ with the neutral singlet is

$$
\left\langle S_{h p} \cdot a \bar{a}|\hat{H}| S_{a b}\right\rangle=2(p h, a b)-(p b, a h),
$$

while the interaction of $T_{h p}^{0} \cdot b \bar{b}$ with the triplet state is

$$
\left\langle T_{h p}^{0} \cdot b \bar{b}|\hat{H}| T_{a b}^{0}\right\rangle=-(p b, a h) .
$$

The final second-order contribution to the singlet-triplet splitting is

$$
J \leftarrow-\frac{8(p h, a b)^{2}-4(p h, a b)[(p a, b h)+(p b, a h)]}{\left(U+\Delta E_{h \rightarrow p}\right)} .
$$

The $a b$ distribution has weak amplitude everywhere and therefore this second order contribution, labeled $\varepsilon_{S E+P}^{2}$ in the work of de Loth et al., ${ }^{31}$ is expected to be small. The corresponding processes are depicted in Diagram 8:
Diagram 8

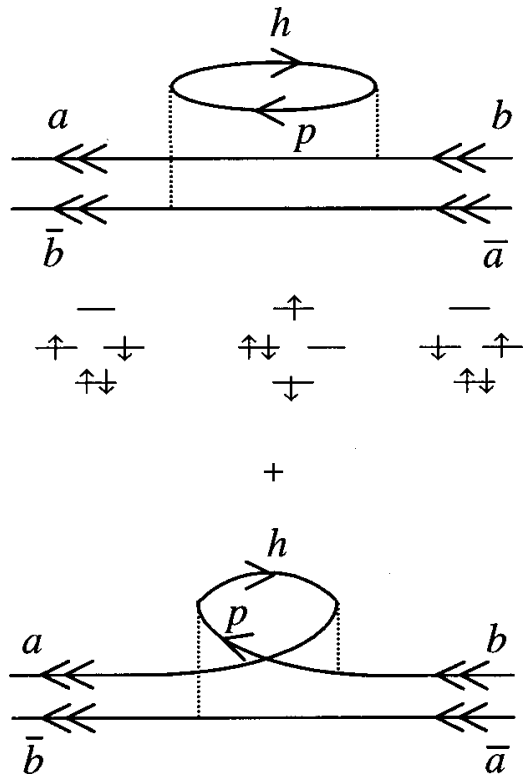

However these outer space $1 h+1 p$ determinants have larger interactions with the ionic VB determinants $|a \bar{a}\rangle$ and $|b \bar{b}\rangle$ which are parts of the valence CI singlet state, $\left|S_{g}\right\rangle[\mathrm{Eq}$. (11)]. Actually,

$$
\frac{1}{\sqrt{2}}\langle(h \bar{p}+p \bar{h}) a \bar{a}|\hat{H}| h \bar{h} a \bar{a}\rangle=\sqrt{2}\left\langle p\left|\hat{h}_{c}+2 \hat{J}_{a}-\hat{K}_{a}\right| h\right\rangle .
$$

The Brillouin's theorem implies that [Eq. (32)],

$$
\left\langle p\left|\hat{h}_{c}+\hat{J}_{a}+\hat{J}_{b}-\frac{\hat{K}_{a}}{2}-\frac{\hat{K}_{b}}{2}\right| h\right\rangle=0,
$$

which gives

$\frac{1}{\sqrt{2}}\langle(h \bar{p}+p \bar{h}) a \bar{a}|\hat{H}| h \bar{h} a \bar{a}\rangle=\sqrt{2}\left\langle p\left|\hat{J}_{a}-\frac{\hat{K}_{a}}{2}-\hat{J}_{b}+\frac{\hat{K}_{b}}{2}\right| h\right\rangle$.

The $\left\langle p\left|\hat{J}_{a}-\hat{J}_{b}\right| h\right\rangle$ integral is large since it represents the coupling between the $h p$ transition distribution with the $A^{-} \cdots A^{+}$valence dipole, resulting from the modification of the field in the ionic VB structure with respect to the mean field. The corresponding energy correction represents the effect of the dynamical polarization of the ligands under the effect of the valence charge fluctuation in the singlet state. The matrix elements may be visualized as in Diagram 9:

Diagram 9
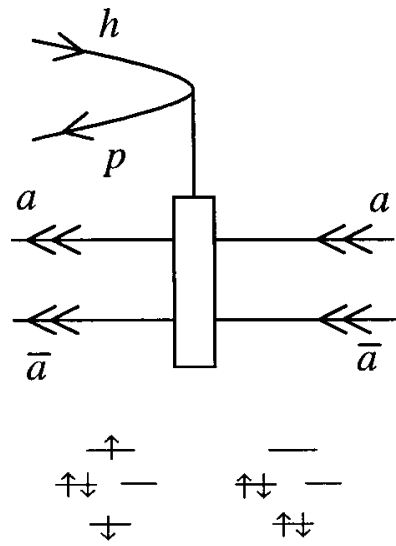
where the vertical box represents the $\left\langle p\left|\widetilde{J}_{a}-\widetilde{J}_{b}\right| h\right\rangle$ integral, $\widetilde{J}_{a}=\hat{J}_{a}-\left(\hat{K}_{a} / 2\right)$ (resp. $\left.\widetilde{J}_{b}\right)$ which corresponds to the instantaneous modification of the field.

In terms of perturbation of the neutral VB determinants, these interactions introduce (a) third-order effects, as represented in Diagram 10:

Diagram 10

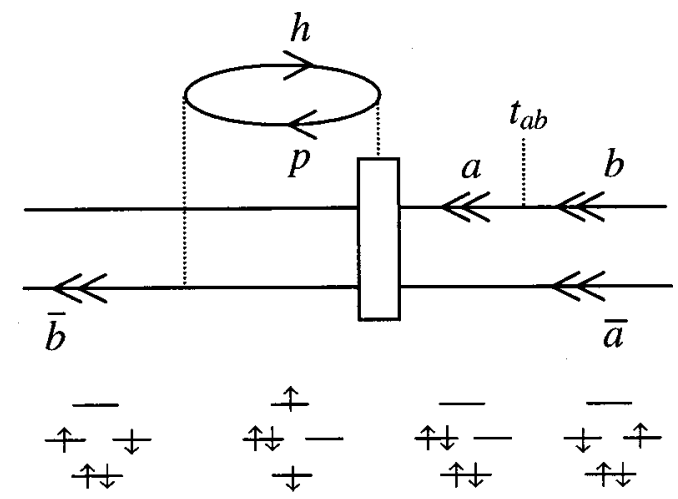

that are expected to be small if the ( $h p, a b)$ integral is small, and (b) fourth-order effects, represented in Diagram 11:

Diagram 11

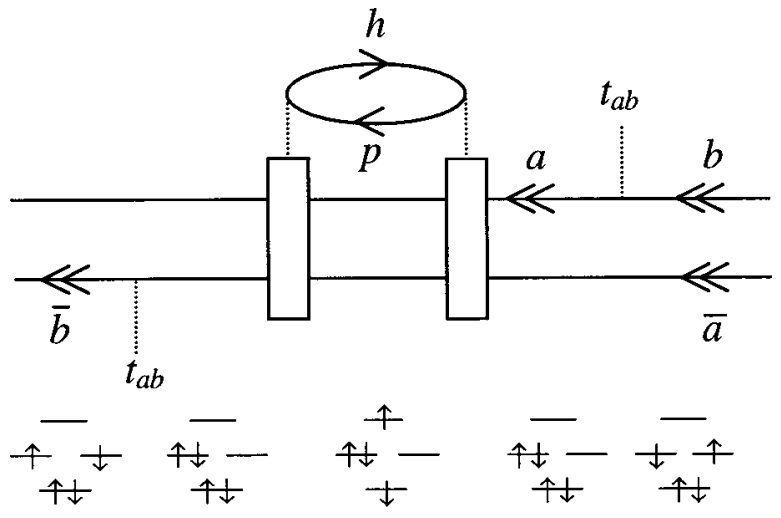

with contributions of the type,

$$
-\frac{t_{a b}\left\langle h\left|\widetilde{J}_{a}-\widetilde{J}_{b}\right| p\right\rangle\left\langle p\left|\widetilde{J}_{a}-\widetilde{J}_{b}\right| h\right\rangle t_{a b}}{U\left(U+\Delta E_{h \rightarrow p}\right) U} .
$$

These fourth-order effects may be considered as contributions to the kinetic exchange term, $t_{a b}^{2} / U$, since

$$
\begin{aligned}
& -\frac{t_{a b}^{2}}{U}-\frac{t_{a b}^{2}}{U^{2}} \sum_{h, p} \frac{\left\langle h\left|\widetilde{J}_{a}-\widetilde{J}_{b}\right| p\right\rangle^{2}}{\left(U+\Delta E_{h \rightarrow p}\right)} \\
& =-\frac{t_{a b}^{2}}{U}\left(1+\sum_{h, p} \frac{\left\langle h\left|\widetilde{J}_{a}-\widetilde{J}_{b}\right| p\right\rangle^{2}}{U\left(U+\Delta E_{h \rightarrow p}\right)}\right) .
\end{aligned}
$$
et ll. $^{31}$

This effect was labeled $\varepsilon_{S E, S E+P}^{4}$ in the work of de Loth

c. The $2 h$ and $2 p$ determinants. The $2 h$ determinants, as represented in Scheme 6, have four electrons in the magnetic orbitals:
Scheme 6

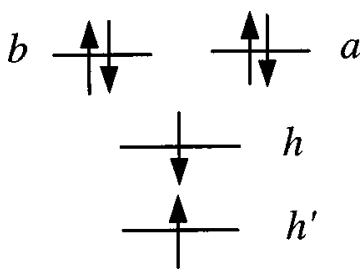

and they contribute to the $\left\langle a \bar{b}\left|\hat{H}_{\mathrm{eff}}^{(2)}\right| b \bar{a}\right\rangle$ effective coupling by

$$
J \leftarrow-\frac{\left(h a, h^{\prime} b\right)\left(h b, h^{\prime} a\right)}{\Delta E_{h h^{\prime} \rightarrow a b}}
$$

as represented in Diagram 12:

Diagram 12

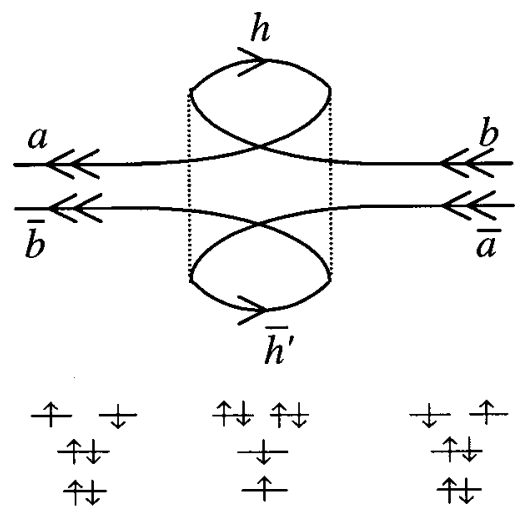

while the $2 p$ determinants have no electrons in the magnetic orbitals, as shown in Scheme 7:

Scheme 7

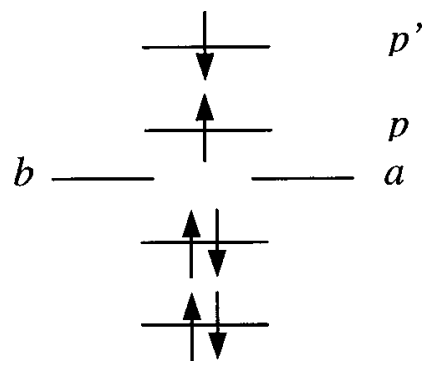

and contribute to the effective coupling by

$$
J \leftarrow-\frac{\left(p b, p^{\prime} a\right)\left(p a, p^{\prime} b\right)}{\Delta E_{a b \rightarrow p p^{\prime}}}
$$

represented in Diagram 13:

Diagram 13

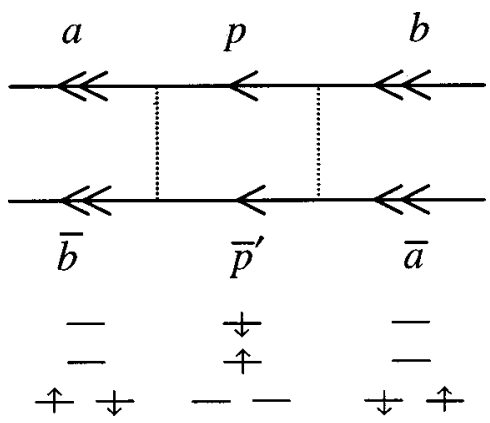


TABLE II. Coupling constant, $J$ (in $\mathrm{cm}^{-1}$ ), at various CI levels, using ROHF orbitals.

\begin{tabular}{lcccc}
\hline \hline & {$\left[\mathrm{Cu}_{2} \mathrm{Cl}_{6}\right]^{-2}$} & {$\left[\mathrm{Cu}_{2}\left(\mathrm{~N}_{3}\right)_{2}\left(\mathrm{NH}_{3}\right)_{6}\right]^{2+}$} & $\mathrm{Cu}_{2}\left(\mathrm{CH}_{3} \mathrm{COO}\right)_{4}\left(\mathrm{H}_{2} \mathrm{O}\right)_{2}$ & $\mathrm{Cu}_{2} \mathrm{O}_{7}$ \\
\hline CASCI & 11 & -82 & -19 & -255 \\
SPCI & 35 & -141 & -36 & -274 \\
DDCI1 & 17 & -362 & -71 & -707 \\
DDCI2 & 9 & -375 & -73 & -744 \\
DDCI & -22 & -802 & -195 & -1077 \\
$J_{\text {exp }}$ & $0,-40^{\mathrm{a}}$ & $<-800^{\mathrm{b}}$ & $-286^{\mathrm{c}},-294 \pm 4^{\mathrm{d}}$ & $-1032 \pm 48^{\mathrm{e}}$ \\
& & & & $-1081 \pm 40^{\mathrm{f}}$ \\
\hline \hline
\end{tabular}

${ }^{\mathrm{a}}$ Reference 62.

${ }^{\mathrm{b}}$ Reference 63.

${ }^{\mathrm{c}}$ Reference 64.
${ }^{\mathrm{d}}$ Reference 38.
${ }^{\text {e }}$ Reference 40.
${ }^{\mathrm{f}}$ Reference 41.
To cause significant contributions it is necessary that both $h$ and $h^{\prime}$ (or $p$ and $p^{\prime}$ ) have important amplitudes near the magnetic centers. The sign of these effects is not predictable in principle. However when $h^{\prime}=h$ (or $p^{\prime}=p$ ) the contributions are necessarily negative and hence favor the singlet state, as evident from the closed shell character of the corresponding perturbers. Therefore these contributions, labeled $\varepsilon_{(L \rightarrow C u)^{2}}^{2}$ and $\varepsilon_{(C u \rightarrow L)^{2}}^{2}$ in Ref. 31, are expected to slightly increase the antiferromagnetic contributions.

\section{Numerical results}

When variational CI calculations are performed instead of perturbative expansions, it is possible to analyze the various physical effects by generating CI spaces of increasing lengths that include different types of determinants:

a first one by generating the $T_{h p}^{+} \cdot T_{a b}^{-}$and $T_{h p}^{-} \cdot T_{a b}^{+}$ determinants, which are responsible for spinpolarization, called SPCI hereafter;

(ii) a larger one that includes all $1 h+1 p$ determinants, usually called first-order CI, hereafter called DDCI1;

(iii) the typical DDCI2 (Ref. 23) list which generates all determinants interacting with both $|a \bar{b}\rangle$ and $|b \bar{a}\rangle$, i.e., all contributing up to the second order, adding the $2 h$ and $2 p$ determinants to the DDCI1 list.

Table II reports the singlet-triplet separation at various
CI levels, for the four systems analyzed. By comparing the different levels, the different physical contributions may be isolated, as indicated in Table III. The CASCI level gives direct exchange and the bare kinetic exchange. The difference between the $J$ value obtained at this level and the $K_{a b}$ obtained from Eq. (20) gives the bare kinetic exchange, according to Eq. (17). The spin polarization is obtained from the difference between the CASCI and the SPCI calculation. Since DDCI1 includes beyond SPCI the effect of the other $1 h+1 p$ determinants, the difference gives their contribution. Finally, DDCI2 also includes $2 h$ and $2 p$ determinants, which effect is isolated from the DDCI2-DDCI1 difference. Looking at Table III several features appear.

First of all, the sign and the magnitude of the spin polarization contribution are variable. This contribution acts in favor of the ferromagnetism $\left(+23 \mathrm{~cm}^{-1}\right)$ in $\left[\mathrm{Cu}_{2} \mathrm{Cl}_{6}\right]^{2-}$ while it is weakly antiferromagnetic in the acetate $(-17$ $\mathrm{cm}^{-1}$ ) and in the cuprate $\left(-19 \mathrm{~cm}^{-1}\right)$. In the azido complex the antiferromagnetic character of this contribution is larger $\left(-59 \mathrm{~cm}^{-1}\right)$. The role of spin polarization had been speculated to be very large ${ }^{35}$ in this complex. It is actually large, but adding this contribution to the valence-only effects (SPCI results) the value of $J$ only reaches $-141 \mathrm{~cm}^{-1}$, five times smaller than the experimental estimate $\left(<-800 \mathrm{~cm}^{-1}\right)$. Additionally, the variational absolute energies at this CI level indicate that the stabilization of both the triplet and the singlet states given by the spin polarization with respect to the valence-only $\mathrm{CI}$ energies is around $-1600 \mathrm{~cm}^{-1}$ in the cu-

TABLE III. Contributions to the coupling constant, $J$ (in $\mathrm{cm}^{-1}$ ), using ROHF orbitals.

\begin{tabular}{lrccr}
\hline \hline & {$\left[\mathrm{Cu}_{2} \mathrm{Cl}_{6}\right]^{2-}$} & {$\left[\mathrm{Cu}_{2}\left(\mathrm{~N}_{3}\right)_{2}\left(\mathrm{NH}_{3}\right)_{6}\right]^{2+}$} & $\mathrm{Cu}_{2}\left(\mathrm{CH}_{3} \mathrm{COO}\right)_{4}\left(\mathrm{H}_{2} \mathrm{O}\right)_{2}$ & $\mathrm{Cu}_{2} \mathrm{O}_{7}$ \\
\hline Direct exchange & 27 & 12 & 4 & 67 \\
Kinetic exchange & -16 & -94 & -23 & -322 \\
Spin polarization & 23 & -59 & -17 & -19 \\
Other $1 h+1 p$ & -18 & -221 & -35 & -432 \\
$2 h, 2 p$ & -8 & -13 & -2 & -36 \\
$2 h+1 p, 1 h+2 p$ & -30 & -427 & -122 & -333 \\
\hline Total & -22 & -802 & -195 & -1077 \\
$J_{\text {exp }}$ & $0,-40^{\mathrm{a}}$ & $<-800^{\mathrm{b}}$ & $-286^{\mathrm{c}},-294 \pm 4^{\mathrm{d}}$ & $-1032 \pm 48^{\mathrm{e}}$ \\
& & & & $-1081 \pm 40^{\mathrm{f}}$ \\
\hline \hline
\end{tabular}

\begin{tabular}{ll}
\hline${ }^{2}$ Reference 62. & ${ }^{\mathrm{d}}$ Reference 38. \\
${ }^{\mathrm{b}}$ Reference 63. & ${ }^{\mathrm{e}}$ Reference 40. \\
${ }^{\mathrm{c}}$ Reference 64. & ${ }^{\mathrm{f}}$ Reference 41.
\end{tabular}


prate and $-1300 \mathrm{~cm}^{-1}$ in the azido complex. This illustrates that the total spin polarization is much larger than its differential effect, as previously discussed in Sec. IV B $1 \mathrm{~b}$.

Second, the effect of the other $1 h+1 p$ contribution is antiferromagnetic and especially large for the azido complex and for the cuprate. Table II shows that the resulting DDCI1 $J$ values are three times larger than the CASCI ones, except for the chloride. The study of the corresponding effective Hamiltonians [paper II (Ref. 58)] confirms that this effect actually comes from the fourth-order mechanism [Diagram 11, Eq. (56)].

Third, the $2 h$ and $2 p$ contributions, i.e., the difference between DDCI2 and DDCI1, give a rather weak $\left(\leqslant 30 \mathrm{~cm}^{-1}\right)$ antiferromagnetic contribution, as speculated above.

Finally the overall result at this stage (DDCI2) is not in satisfactory agreement with experiment: for the azido and for the acetato complexes only $30 \%-40 \%$ of the final value is reached; for the perovskite fragment the result is only slightly better, since $70 \%$ of the experimental value is reached. This $70 \%$ ratio was observed on a wide series of perovskites of both $\mathrm{Cu}$ and $\mathrm{Ni}^{59-61}$ Finally, the sign remains incorrect for the $\left[\mathrm{Cu}_{2} \mathrm{Cl}_{6}\right]^{2-}$ complex. Hence some important physical effect is lacking at this level.

\section{Enlarging the model space to the whole valence space and adding $2 h+1 p$ and $1 h+2 p$ outer space determinants}

\section{Theory}

The preceding list of perturbers (the DDCI2 list) was obtained from second order arguments taking the neutral VB forms as degenerate model space. Since the ionic VB forms are expected to play a crucial role in the kinetic exchange, the next improvement consists in enlarging the model space from the neutral VB forms only to the full valence space. The new model space is no longer degenerate. Then at the second-order perturbative level all the determinants $|\beta\rangle$ that interact with either neutral or ionic VB determinants must be added to the DDCI2 list. Most of them, obtained from purely inactive $h h \rightarrow p p^{\prime}$ excitations, i.e., $2 h+2 p$ determinants, result in a common shift of all the diagonal matrix elements. They can therefore be omitted for the calculation of energy differences as argued in the proposal of the DDCI (Ref. 22) method and the only determinants to be added to the DCCI2 space are the $2 h+1 p$ and $1 h+2 p$ ones.

a. $2 h+1 p$ determinants: The antiferromagnetic character of their contribution and their impact on the wave function. In the recent past ${ }^{28}$ the role of this type of excitations has been shown to be very important in the evaluation of $J$, to which it brings a large antiferromagnetic contribution. Among them, a special subset of determinants has been shown to play a leading role, namely the single $h \rightarrow p$ excitations on the ligand to metal $l \rightarrow a, b$ charge transfer configurations. Therefore special attention is paid to the $2 h$ $+1 p$ determinants, that have three electrons in the magnetic orbitals, as shown in Scheme 8:
Scheme 8

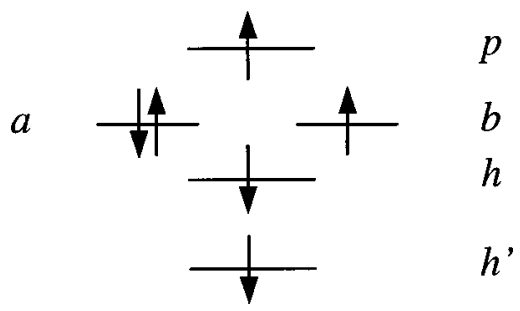

and lead to second order corrections to the effective hopping integral between neutral and ionic states according to

$$
\begin{aligned}
t_{a b}^{\mathrm{eff}} & =\left\langle a \bar{b}\left|\hat{H}_{\mathrm{eff}}^{(2)}\right| a \bar{a}\right\rangle \\
& =\langle a \bar{b}|\hat{H}| a \bar{a}\rangle+\sum_{\beta \notin S} \frac{\langle a \bar{b}|\hat{H}| \beta\rangle\langle\beta|\hat{H}| a \bar{a}\rangle}{E_{0}^{(0)}-E_{\beta}^{(0)}}=t_{a b}+\Delta t_{a b} .
\end{aligned}
$$

The specific contribution to Eq. (59) of $2 h+1 p$ determinants is represented in Diagram 14:

Diagram 14

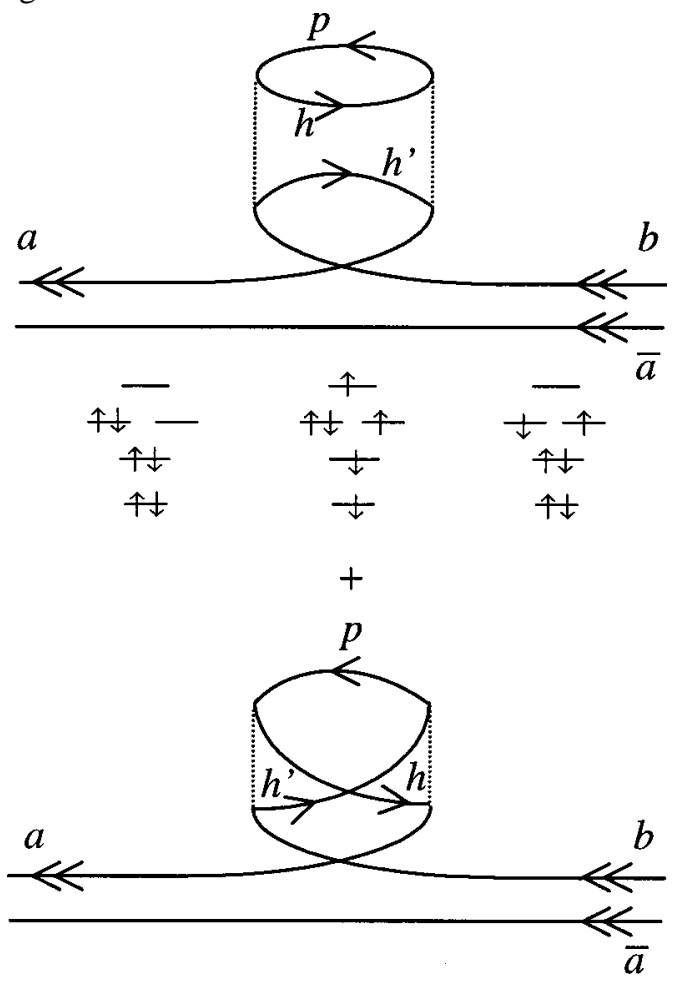

The analytic contribution to $\Delta t_{a b}$ is

$\Delta t_{a b} \leftarrow \frac{2\left(h p, a h^{\prime}\right)\left(b h^{\prime}, h p\right)-\left(h^{\prime} p, a h\right)\left(b h^{\prime}, h p\right)}{E_{0}^{(0)}-E_{\beta}^{(0)}}$.

For a non-negligible contribution from the second term both $h$ and $h^{\prime}$ must have important amplitudes near $A$ and $B$. It is likely to be less important than the first one which only requires $h^{\prime}$ to be important near $A$ and $B$, i.e., to be an orbital of the bridging ligand(s). The distributions $a h^{\prime}$ and $b h^{\prime}$ may be both important dipolar distributions when $h^{\prime}=l$, i.e., the doubly occupied orbital(s) of the bridging-ligand having large delocalization tails on both metallic centers. Each of these elementary excitations, according to Diagram 15: 
Diagram 15

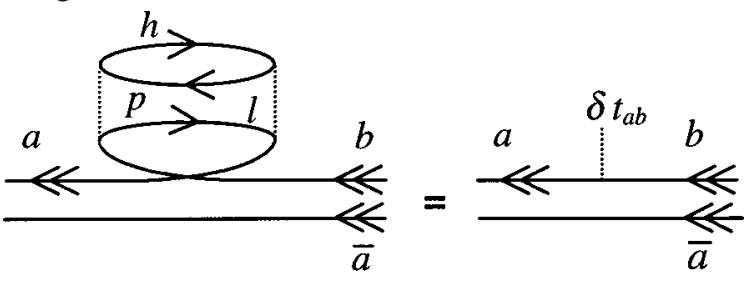

$$
\begin{array}{ccc}
\bar{\uparrow} & \uparrow & - \\
\uparrow \downarrow \uparrow & \downarrow \uparrow \\
\uparrow \downarrow & \downarrow & \uparrow \downarrow \\
\uparrow \downarrow & \downarrow & \uparrow \downarrow
\end{array}
$$

results in a modification of the $t_{a b}$ hopping integral,

$$
\delta t_{a b}=\frac{2(h p, a l)(b l, h p)}{\Delta E_{C T}+\Delta E_{h \rightarrow p}} .
$$

Therefore, the global $2 h+1 p$ contribution to $t_{a b}^{\mathrm{eff}}$ are governed by

$$
\Delta t_{a b}=\sum_{l} \sum_{h} \sum_{p} \frac{2(h p, a l)(b l, h p)}{\Delta E_{C T}+\Delta E_{h \rightarrow p}},
$$

which involves coupling of $h p$ transition dipoles of the surrounding electrons with the transition dipoles $a l$ and $b l$, as shown in Diagram 15. This $\Delta t_{a b}$ modification of the interaction between neutral and ionic determinants only affects the singlet state since the ionic determinants being pure singlets do not contribute to the triplet state wavefunction. This effect results in third and fourth-order contributions to $J$, represented in Diagrams 16 and 17, respectively:

Diagram 16

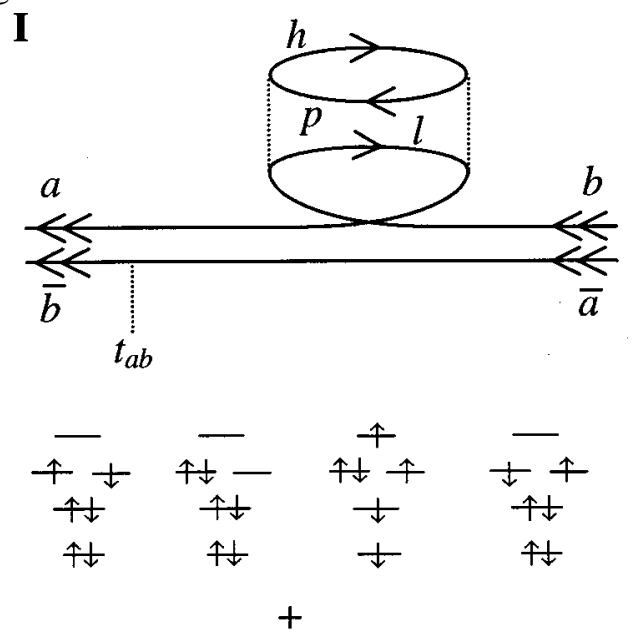

II

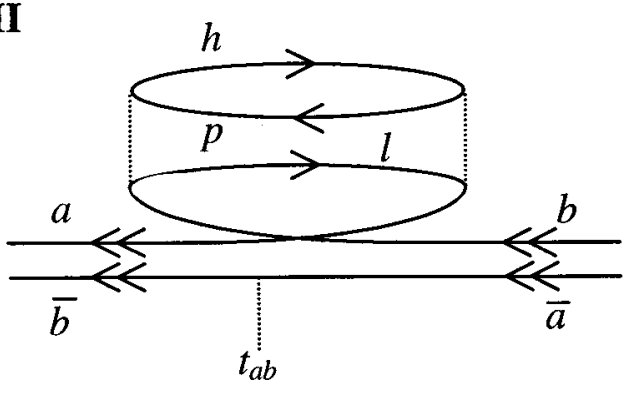

Diagram 17

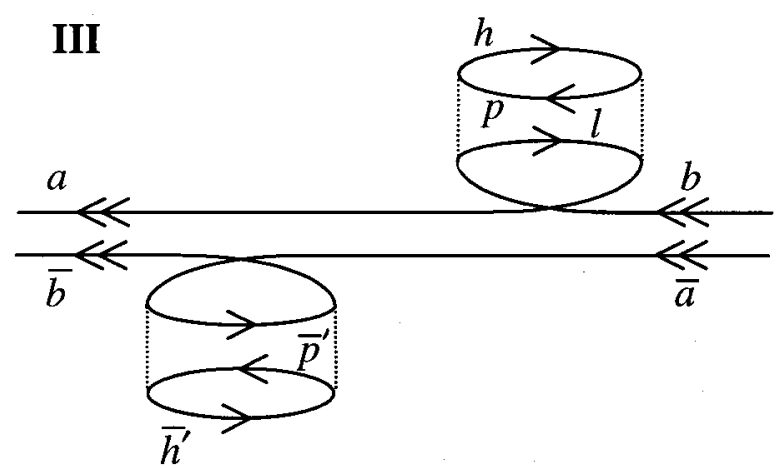

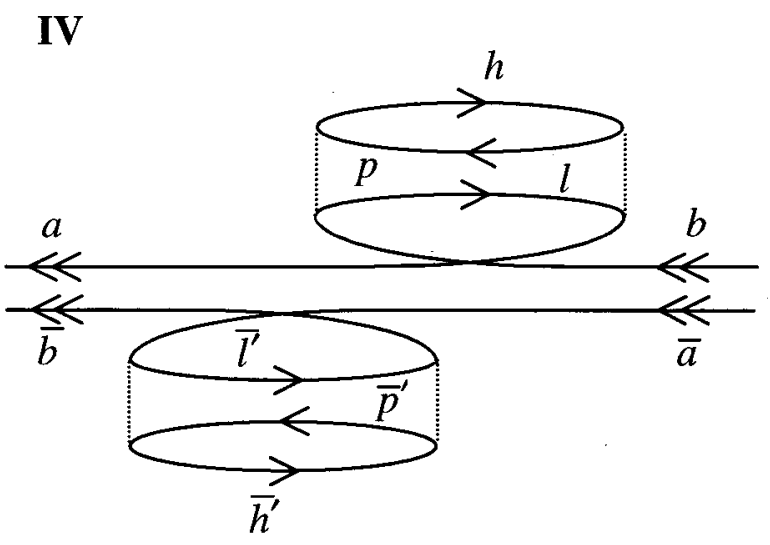

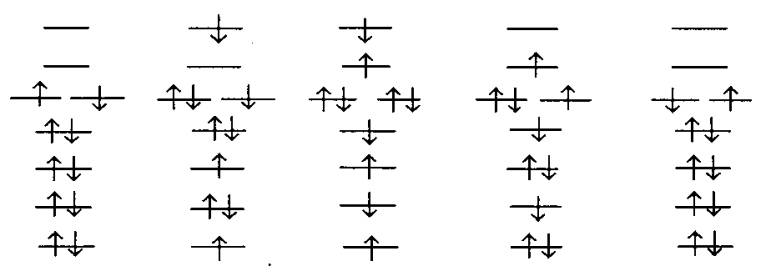

Among these diagrams, I and III should be the leading ones, since they involve lower energy denominators and have weaker exclusion requirements. From Diagram 15 and Eq. (61) it is possible to write these contributions to $J$ as

$$
\begin{aligned}
J \leftarrow & \underbrace{-\frac{4 t_{a b} \delta t_{a b}}{U}-\frac{2 t_{a b} \delta t_{a b}}{\Delta E_{C T}+\Delta E_{h \rightarrow p}}}_{\text {3rd order }} \\
& \underbrace{-\frac{4 \delta t_{a b}^{2}}{U}-\frac{8 \delta t_{a b}^{2}}{2 \Delta E_{C T}+\Delta E_{h \rightarrow p}+\Delta E_{h^{\prime} \rightarrow p^{\prime}}}}_{4 \text { th order }} .
\end{aligned}
$$

In order to analyze the sign of the $2 h+1 p$ determinant contribution, it is useful to introduce strongly localized orbitals, $\underline{a}, \underline{b}, \underline{l}$, on the metal and on the bridging ligand such as 


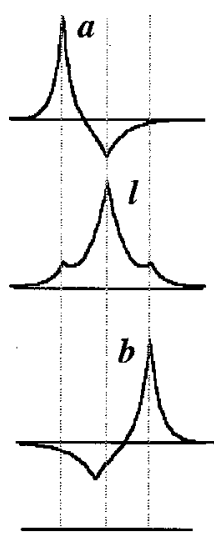

A L B

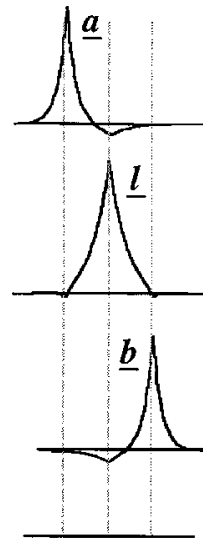

A L B

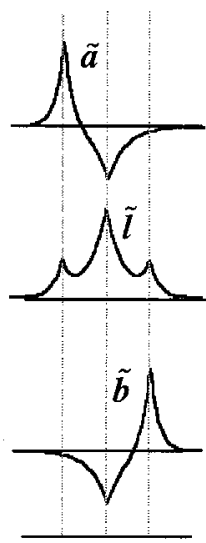

A L B a)

b)

c)

FIG. 4. Schematic shape of different types of magnetic orbitals obtained by rotation of symmetry adapted MOs. (a) ROHF orbitals; (b) strongly localized orbitals; (c) natural orbitals.

those used in the Anderson model. They can be expressed as linear combinations of the ROHF orbitals, $a, b$, and $l$, with $a$ and $b$ the magnetic orbitals obtained by rotation of the symmetry adapted ones [cf. Eq. (2)], and $l$ the orbitals with a dominant weight on the bridging-ligand. Both sets are related by the localizing transformation, $\{\underline{a}, \underline{b}, \underline{l}\}=U\{a, b, l\}$. To the first order, the linear combination gives

$$
\begin{array}{lll}
\underline{a}=a+\lambda l & & a=\underline{a}-\lambda \underline{l} \\
\underline{l}=l-\lambda(a+b) & \text { or } & l=\underline{l}+\lambda(\underline{a}+\underline{b}) \\
\underline{b}=b+\lambda l & & b=\underline{b}-\lambda \underline{l} .
\end{array}
$$

$\lambda$ is positive since in the ROHF orbitals the ligand orbital, $l$, takes in-phase tails on the magnetic centers and, consequently, the magnetic orbitals $a$ and $b$ take out-of-phase tails on the ligand. Figure 4 illustrates schematically the transformation from the ROHF orbitals to these new more localized orbitals.

The $\underline{a}, \underline{b}$, and $\underline{l}$ orbitals are no longer optimal since doubly and singly occupied MOs have been mixed and the new reference configurations,

$$
\begin{aligned}
& T_{\underline{a} \underline{b}}^{0}=\left|\frac{1}{\sqrt{2}} \cdots \underline{l} \underline{\underline{l}} \cdots(\underline{a} \underline{\bar{b}}-\underline{b} \underline{\bar{a}})\right\rangle, \\
& S_{\underline{a} \underline{b}}=\left|\frac{1}{\sqrt{2}} \cdots \underline{l} \underline{\underline{\underline{I}}} \cdots(\underline{a} \underline{\bar{b}}+\underline{b} \underline{\bar{a}})\right\rangle,
\end{aligned}
$$

no longer satisfy the Brillouin's theorem. In particular,

$\left\langle a_{\underline{a}}^{+} a_{\underline{\underline{l}}} \cdots \underline{l} \underline{\underline{\bar{l}}} \cdots(\underline{a} \underline{\bar{b}}-\underline{b} \underline{\bar{a}})|\hat{H}| \cdots \underline{\underline{l}} \underline{\bar{l}} \cdots(\underline{a} \underline{\bar{b}}-\underline{b} \underline{\bar{a}})\right\rangle=t_{\underline{a} \underline{l}}$

is no longer null and induces important coefficients on the ligand to metal charge transfer configurations.

Let us now analyze the sign of the contribution of the $2 h+1 p$ determinants through these strongly localized orbitals, $\underline{a}, \underline{b}$, and $\underline{l}$. In the $A-L-B$ structure, it is easy to demonstrate that the distributions $a l$ and $b l$ can be approximated by

$$
a l \approx \lambda\left(\underline{a}^{2}-\underline{l}^{2}\right) \text { and } b l \approx \lambda\left(\underline{b}^{2}-\underline{l}^{2}\right) .
$$

The two $a l$ and $b l$ dipolar distributions have opposite directions. In order to have a significant contribution to $t_{a b}^{\mathrm{eff}}$ in Eq. (61) $h$ and $p$ have to be located on the bridge and in this case the $h p$ transition dipole interacts with opposite signs with the $a l$ and $b l$ dipoles. Consequently $\delta t_{a b}$ is negative, i.e., has the same sign as $t_{a b}$. Hence, the $2 h+1 p$ mechanism acts as an enhancement of $t_{a b}$, as numerically confirmed in paper $\Pi,{ }^{58}$ and following Eq. (63), the $2 h+1 p$ determinants induce an increase of the $J$ value.

The precedent discussion leads to a modification of the physical interpretation previously proposed by us in recent papers $^{28}$ to explain the role of the $2 h+1 p$ excitations. The interpretation was based on the two-band model. We suggested that the $2 h+1 p$ determinants introduce the dynamical polarization of the inactive electrons in the field created by the LMCT states. When looking at Diagram 4, this dynamical polarization would be brought by $h p$ single excitations after the LMCT processes. This single excitation introduces the effect of the modification of the electric field in the LMCT state, analogous to the effect occurring in the ionic valence states shown in Diagram 11. It gives rise to Diagram 18:

\section{Diagram 18}
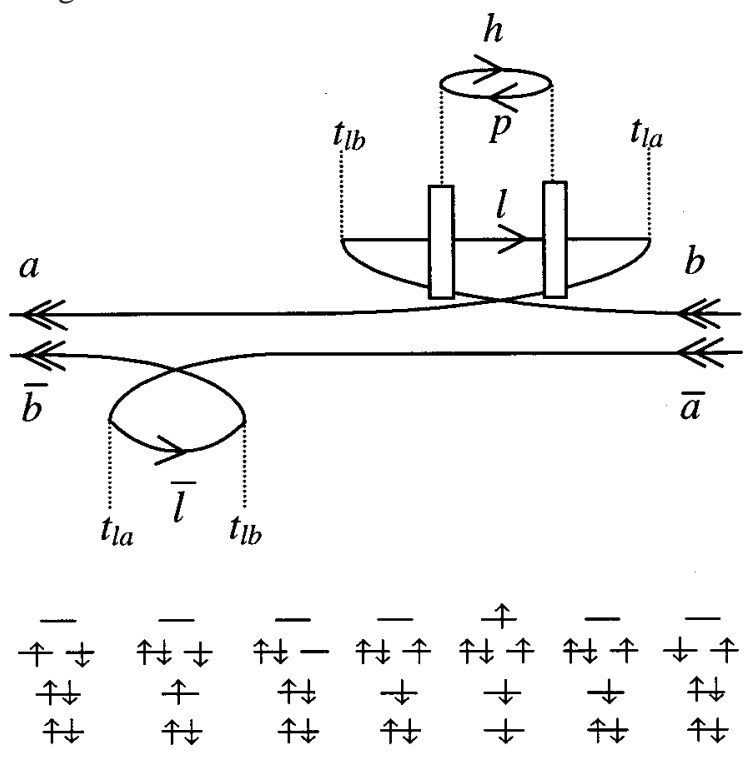

where the box represents the matrix element $\left\langle h\left|\widetilde{J}_{a}-\widetilde{J}_{l}\right| p\right\rangle$.

The interpretation was based on the assumption that the $2 h+1 p$ configurations were reducing the $\Delta E_{\mathrm{CT}}$ energy appearing in Eqs. 23 and 24 and the observed increase of the weight of the LMCT configurations in the wave function seemed to confirm our interpretation; ${ }^{28}$ see Table IV ROHF column.

Diagram 18 involves $t_{l a}$ and $t_{l b}$ hopping integrals which are not null when strongly localized orbitals, $\underline{a}, \underline{b}$, and $\underline{l}$, are used. In this case the leading effect goes through Diagram 18 and the interpretation of the effect as due to the polarization of the charge transfer states is correct. This is no longer the case when ROHF orbitals are used since then $t_{l a}=t_{l b}=0$ and Diagram 18 has an almost null contribution to the coupling, as discussed above. The mechanism is not a 6th order one (Diagram 18) but third (Diagram 16) and fourth-order ones 
TABLE IV. Largest ligand to metal charge transfer coefficients in $\mathrm{Cu}_{2} \mathrm{O}_{7}$ cluster for the singlet and triplet states, at DDCI1 and DDCI levels, using either ROHF or natural orbitals. $L_{g}$ and $L_{u}$ represent ligand-centered MOs with $g$ and $u$ symmetry, respectively.

\begin{tabular}{ccccc}
\hline \hline & & ROHF MOs & Natural MOs \\
\hline \multirow{2}{*}{ DDCI1 } & Singlet & $L_{u} \rightarrow u$ & 0.0292 & 0.0826 \\
& & $L_{g} \rightarrow g$ & 0.0053 & 0.0731 \\
& Triplet & $L_{g} \rightarrow u$ & 0.0167 & 0.0917 \\
& & $L_{u} \rightarrow g$ & 0.0139 & 0.0684 \\
DDCI & Singlet & $L_{u} \rightarrow u$ & 0.1249 & 0.0396 \\
& & $L_{g} \rightarrow g$ & 0.0644 & 0.0176 \\
& Triplet & $L_{g} \rightarrow u$ & 0.1030 & 0.0223 \\
& & $L_{u} \rightarrow g$ & 0.0829 & 0.0338 \\
\hline \hline
\end{tabular}

(Diagram 17). The observed increase of the weight of LMCT states $^{28}$ from DDCI1 or DDCI2 calculations to DDCI, see Table IV, can be interpreted as a second order contribution to the wave function depicted in Diagram 19:

Diagram 19

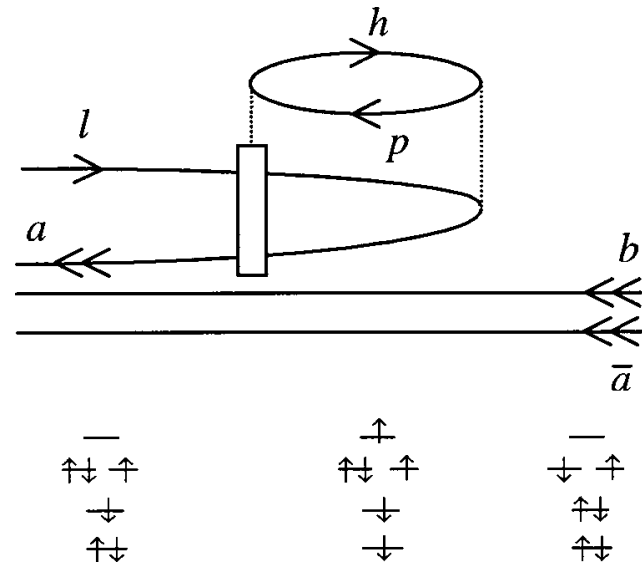

In contrast to Diagram 18 , the $2 h+1 p$ state is obtained in a unique step, i.e., the charge transfer and the polarization occur simultaneously in the right part of Diagrams 16 and 17. The second order coefficient of the LMCT determinant, $|a \bar{l} b \bar{a}\rangle$, is

$$
\begin{aligned}
c_{l \rightarrow a}^{(2)} & =\sum_{h, p} \frac{\langle h \bar{h} a \bar{l} b \bar{a}|\hat{H}| p \bar{h} a \bar{l} b \bar{a}\rangle\langle p \bar{h} a \bar{l} b \bar{a}|\hat{H}| h \bar{h} l \bar{l} b \bar{a}\rangle}{\Delta E_{C T}\left(\Delta E_{C T}+\Delta E_{h \rightarrow p}\right)} \\
& =\sum_{h, p} \frac{\left\langle h\left|\hat{J}_{a}-\hat{J}_{l}\right| p\right\rangle(h p, l a)}{U\left(U+\Delta E_{h \rightarrow p}\right)} .
\end{aligned}
$$

Using relation (67) gives

$$
\begin{aligned}
(h p, l a) & =\lambda[(h p, \underline{a} \underline{a})-(h p, \underline{l} \underline{l})] \\
& \cong \lambda\left\langle h\left|\hat{J}_{\underline{a}}-\hat{J}_{\underline{l}}\right| p\right\rangle \cong \lambda\left\langle h\left|\hat{J}_{a}-\hat{J}_{l}\right| p\right\rangle .
\end{aligned}
$$

Hence the second order coefficient of the determinant,

$$
c_{l \rightarrow a}^{(2)} \cong \lambda \sum_{h, p} \frac{\left\langle h\left|J_{a}-J_{l}\right| p\right\rangle^{2}}{U\left(U+\Delta E_{h \rightarrow p}\right)}
$$

is positive since $\lambda$ is positive, as shown before. A direct consequence of the sign of this coefficient is the increase of the metal-ligand delocalization observed in the natural orbitals, when comparing them to the ROHF ones, as shown elsewhere. $^{57}$
Mixing the reference determinant $|l \bar{l} a \bar{b}\rangle$ with $c_{l \rightarrow a}^{(2)}|a \bar{l} b \bar{a}\rangle$ is equivalent to mix the ROHF orbitals, $a$ and $l$, to generate $\tilde{l}=l+c_{l \rightarrow a}^{(2)}(a+b)$ and $\tilde{a}=a-c_{l \rightarrow a}^{(2)} l$ natural orbitals, as shown in Fig. 4(c). By using Eq. (64), the natural orbitals may be expressed as

$$
\tilde{l}=\left(1-c_{l \rightarrow a}^{(2)} \lambda\right) \underline{l}+\left(\lambda+c_{l \rightarrow a}^{(2)}\right)(\underline{a}+\underline{b})
$$

and

$$
\widetilde{a}=\left(1-c_{l \rightarrow a}^{(2)} \lambda\right) \underline{a}-\left(\lambda+c_{l \rightarrow a}^{(2)}\right) \underline{l}
$$

which explains the larger ligand/metal delocalization found in natural orbitals.

b. $1 h+2 p$ determinants. The $1 h+2 p$ determinants have only one electron in the active orbitals, as represented in Scheme 9:

Scheme 9

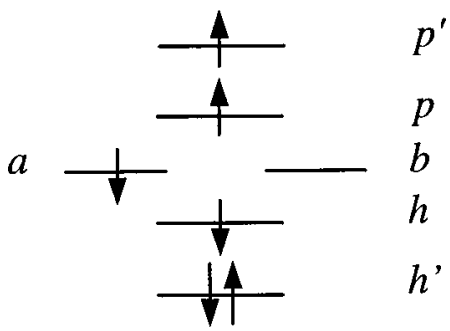

and lead to second order corrections to the effective coupling between neutral and ionic states as shown in Diagram 20:

Diagram 20
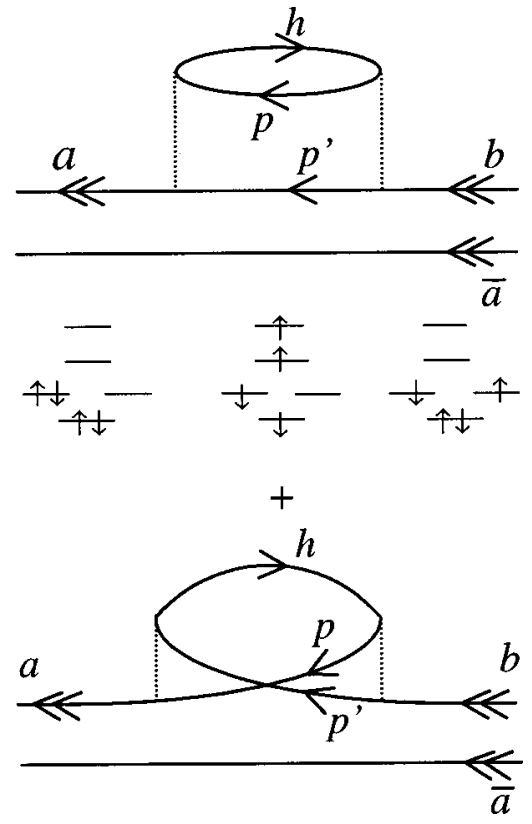

giving

$\Delta t_{a b} \leftarrow \frac{2\left(h p, a p^{\prime}\right)\left(b p^{\prime}, h p\right)-\left(p^{\prime} h, a p\right)\left(b p^{\prime}, h p\right)}{E_{0}^{(0)}-E_{\beta}^{0}}$.

The most important contributions arise from the first term when $p^{\prime}$ is a bridging-ligand antibonding orbital, with large $a p^{\prime}$ and $b p^{\prime}$ distributions. In this case the intermediate state involves a simultaneous $M \rightarrow L$ charge transfer and a single excitation in the environment. These processes lead again to third and fourth order corrections to $J$, which take the same 
form as Eq. (63), such as represented in Diagrams 21 and 22, respectively:

Diagram 21

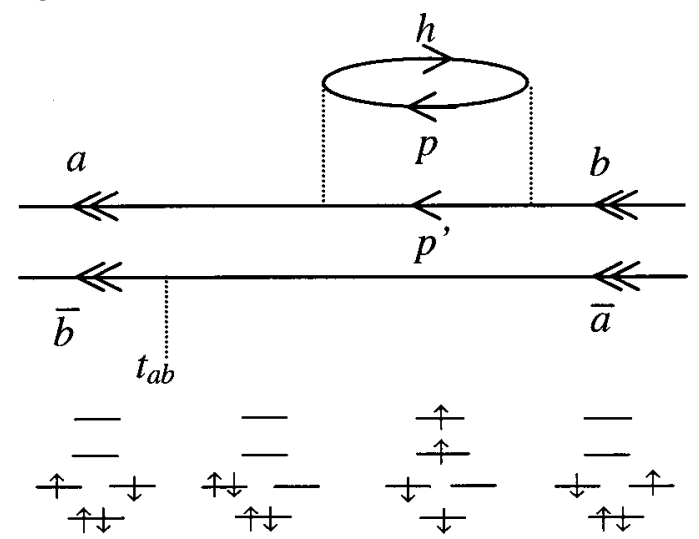

Diagram 22
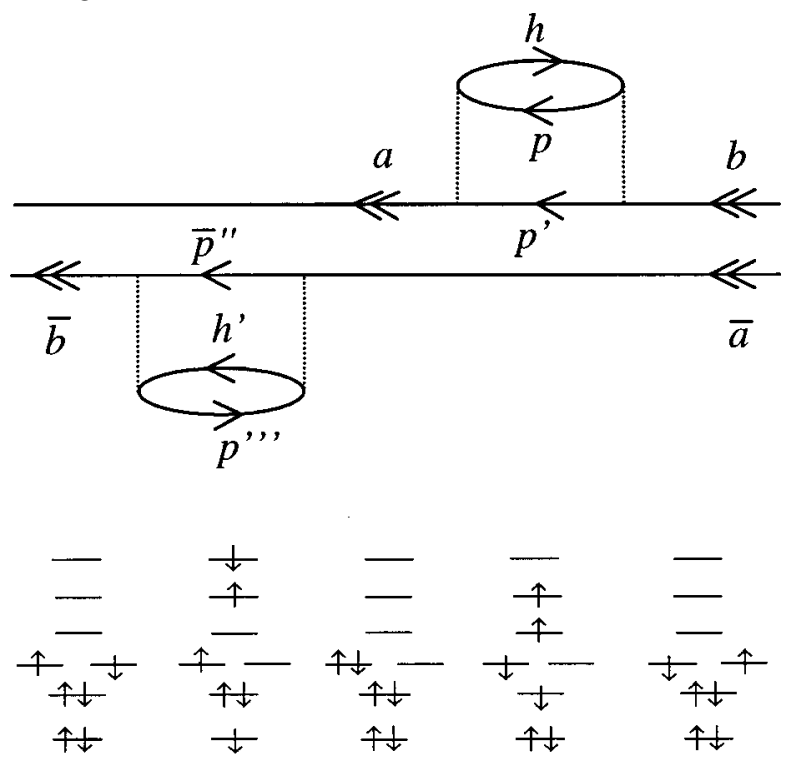

These contributions of $1 h+2 p$ and $2 h+1 p$ determinants are nonadditive contributions since mixed fourth-order terms also exists, such as represented in Diagram 23:

Diagram 23
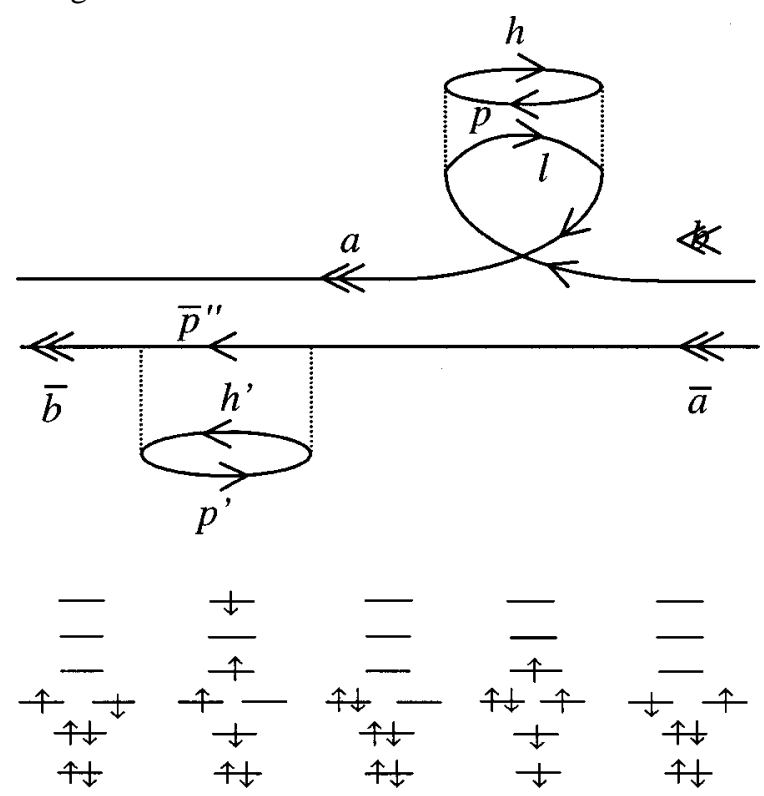

Adding the $2 h+1 p$ and $1 h+2 p$ determinants to the DDCI2 list leads to the full DDCI space and has been shown in the recent past ${ }^{26-28,60,61}$ to provide a systematic agreement with experiment, in all the systems studied. The effect of the $1 \mathrm{~h}$ $+2 p$ determinants goes through a modification of $t_{a b}$ involving products of two bielectronic integrals, each of them corresponding to the interaction of two transition dipoles. The bielectronic integral $\left(a p^{\prime}, h p\right)\left[\operatorname{resp} .\left(b p^{\prime}, h p\right)\right]$ is involved in the dispersion energy between the electrons in $a$ (resp. $b$ ) and in $h$, which is proportional to $\left(a p^{\prime}, h p\right)^{2}$ [resp. $\left.\left(b p^{\prime}, h p\right)^{2}\right]$. The contribution to the effective hopping integrals no longer involves the quadratic terms, but a crossed product $\left(a p^{\prime}, h p\right)\left(b p^{\prime}, h p\right)$. This contribution may therefore be considered of dispersive origin. A similar physical content can be attributed to the $2 h+1 p$ processes, now involving al transition dipoles.

The effect of $2 h+1 p$ and $1 h+2 p$ can therefore be considered as a dispersive contribution to the kinetic exchange. In other terms, the polarizability of the spectator electrons of the ligand helps to transfer the active electrons from one magnetic site to the other. It is expected that the more polarizable the bridging-ligand, the larger this effect will be.

\section{Numerical results}

When comparing DDCI2 and DDCI results in Table II it appears that the results are significantly improved over DDCI2. The $\left[\mathrm{Cu}_{2} \mathrm{Cl}_{6}\right]^{2-}$ complex is now weakly antiferromagnetic, in accordance with some experimental data. ${ }^{62}$ Moreover, in all cases a reasonable quantitative agreement with experiment is obtained now. When looking at the isolated contribution of $2 h+1 p$ and $1 h+2 p$ determinants, reported in Table III, several features can be commented.

Table III shows that the overall effect of these determinants in the systems studied is systematically antiferromagnetic. Additional calculations adding only the $2 h+1 p$ or the $1 h+2 p$ to DDCI2 have been performed on the $\mathrm{CuO}_{2}$ lattice fragment and on the copper acetate. They show that:

(i) the $2 h+1 p$ contribution is large and antiferromagnetic (leading to an exaggerated value of $J=-1532$ $\mathrm{cm}^{-1}$ in the cuprate and $-284 \mathrm{~cm}^{-1}$ in the acetate); the $1 h+2 p$ contribution is ferromagnetic (reducing $J$ to $-563 \mathrm{~cm}^{-1}$ in the cuprate and to $-62 \mathrm{~cm}^{-1}$ in the acetate);

(iii) they interact at fourth order, as previously noticed (cf. Diagram 23) since the final contribution is not the sum of the separate contributions.

The role of the bridging-ligand polarizability is manifest. The relative effect of the dispersive contribution to the total value of $J$ is much larger for the azido and the acetato ligands (where they multiply $J$ by a factor close to 3 ) than for the oxo bridge ( $30 \%$ increase of $J$ ). The chloride case is exceptional since the effect changes the sign of an overall very small $J$.

\section{USE OF NATURAL ORBITALS}

Natural orbitals obtained from diagonalization of the one-electron density matrix, calculated from the DDCI most 
TABLE V. Contributions to the coupling constant, $J$ at the CASCI level, using quasinatural orbitals. Direct exchange, $K_{a b}$, hopping integral, $t_{a b}$, on-site repulsion, $U$. All results in $\mathrm{cm}^{-1}$.

\begin{tabular}{lcccc}
\hline \hline & {$\left[\mathrm{Cu}_{2} \mathrm{Cl}_{6}\right]^{2-}$} & {$\left[\mathrm{Cu}_{2}\left(\mathrm{~N}_{3}\right)_{2}\left(\mathrm{NH}_{3}\right)_{6}\right]^{2+}$} & $\mathrm{Cu}_{2}\left(\mathrm{CH}_{3} \mathrm{COO}\right)_{4}\left(\mathrm{H}_{2} \mathrm{O}\right)_{2}$ & $\mathrm{Cu}_{2} \mathrm{O}_{7}$ \\
\hline $2 K_{a b}$ & 162 & 720 & 66 & 334 \\
$2 t_{a b}$ & -3528 & -12200 & -3992 & -11179 \\
$U\left(\times 10^{-4}\right)$ & 14.8 & 15.2 & 16.0 & 15.8 \\
$J$ & +78 & -253 & -33 & -452 \\
$J_{\text {exp }}$ & $0,-40^{\mathrm{a}}$ & $<-800^{\mathrm{b}}$ & $-286^{\mathrm{c}},-294 \pm 4^{\mathrm{d}}$ & $-1032 \pm 48^{\mathrm{e}}$ \\
& & & & $-1081 \pm 40^{\mathrm{f}}$ \\
\hline
\end{tabular}

${ }^{\mathrm{a}}$ Reference 62.

${ }^{\mathrm{b}}$ Reference 63.

${ }^{\mathrm{c}}$ Reference 64.

exact wave function can be used to reanalyze the problem. To apply the difference dedicated technique, a common set of MOs is to be used for both states, and therefore mean natural orbitals have been calculated from the average of the singlet and triplet states density matrices. As rationalized in Sec. IV of the present work, the delocalization tails between the ligand and the metallic centers become larger in the natural orbitals than in the ROHF ones, and therefore the $a b$ overlap distribution is expected to have larger amplitudes, especially on the bridging-ligand. The remaining parameters should be modified in consequence: the direct exchange, $K_{a b}$, is expected to be enlarged as well as the hopping integral, $\left|t_{a b}\right|$, and the on-center effective repulsion, $U$, should be slightly reduced. The results in Table $\mathrm{V}$ by comparing with Table I show that

$K_{a b}$ is drastically increased, by a factor 5 in $\left[\mathrm{Cu}_{2} \mathrm{Cl}_{6}\right]^{2-}$ and $\mathrm{Cu}_{2} \mathrm{O}_{7}$, by 20 in the acetate, by $60 \mathrm{in}$ the azido complex;

(ii) $\left|t_{a b}\right|$ is larger, multiplied by 2 in $\left[\mathrm{Cu}_{2} \mathrm{Cl}_{6}\right]^{2-}$ and in the acetate, by 1.4 in $\mathrm{Cu}_{2} \mathrm{O}_{7}$, and by 3 in the azido complex;

(iii) $U$ is reduced by $20 \%-25 \%$ in all the systems.

The resulting valence-only CI remains unreliable. It is wrong in $\left[\mathrm{Cu}_{2} \mathrm{Cl}_{6}\right]^{2-},+78 \mathrm{~cm}^{-1}$ (incorrect sign), and weakly improved in $\mathrm{Cu}_{2} \mathrm{O}_{7},-452 \mathrm{~cm}^{-1}$ (instead $-255 \mathrm{~cm}^{-1}$ ), more significantly in the azido complex, -253 instead of -82 $\mathrm{cm}^{-1}$, and -33 instead of $-19 \mathrm{~cm}^{-1}$ in the acetate. There is no reason to hope that the complex dynamical correlation effects can be kept through a mere revision of the orbitals. It is interesting to repeat the DDCI1, DDCI2, and DDCI calcu-

\author{
${ }^{\mathrm{d}}$ Reference 38 . \\ ${ }^{\mathrm{e}}$ Reference 40. \\ ${ }^{\mathrm{f}}$ Reference 41.
}

lations with these orbitals. Comparing Tables II and VI one sees that the DDCI1 and DDCI2 results are significantly improved, i.e., closer to the final value. The final (DDCI) results are quite comparable to those obtained from ROHF orbitals, but in slightly better agreement with experiment.

The $2 h+1 p$ and $1 p+2 h$ introduced in DDCI have now a much weaker effect, as shown in Table VII $\left(+6 \mathrm{~cm}^{-1}\right.$ instead $-30 \mathrm{~cm}^{-1}$ for ROHF orbitals in $\left[\mathrm{Cu}_{2} \mathrm{Cl}_{6}\right]^{2-},-185$ $\mathrm{cm}^{-1}$ instead of $-333 \mathrm{~cm}^{-1}$ in the $\mathrm{Cu}_{2} \mathrm{O}_{7}$ fragment, -310 $\mathrm{cm}^{-1}$ instead of $-427 \mathrm{~cm}^{-1}$ in the azido complex and practically invariant in the acetate). In the azido complex these effects now increase $J$ by $38 \%$ instead of $114 \%$ when using ROHF orbitals. Their effect still doubles the value of $J$ for the acetate, while it multiplies it by a factor 3 when using ROHF orbitals. These two last cases show that it is not possible to rely on the use of quasinatural orbitals to rest on the DDCI2 level of calculation, omitting the $2 h+1 p$ and $1 h$ $+2 p$ excitations. One may actually observe that the coefficients of the ligand to metal charge transfer configurations are no longer zero at the $1 h+1 p$ or the DDCI2 level, cf. Table IV, since the Brillouin's theorem is not satisfied anymore. However these coefficients are decreased when the $2 h+1 p$ and $1 h+2 p$ configurations are involved. This is opposite to the phenomenon observed when starting from ROHF orbitals.

\section{CONCLUSIONS}

Recent works have shown the possibility to obtain accurate values of the magnetic coupling constant through $a b$ initio CI calculations, especially when using the difference

TABLE VI. Coupling constant, $J$ (in $\mathrm{cm}^{-1}$ ), at various CI levels using quasinatural orbitals.

\begin{tabular}{lcccc}
\hline \hline & {$\left[\mathrm{Cu}_{2} \mathrm{Cl}_{6}\right]^{2-}$} & {$\left[\mathrm{Cu}_{2}\left(\mathrm{~N}_{3}\right)_{2}\left(\mathrm{NH}_{3}\right)_{6}\right]^{2+}$} & $\mathrm{Cu}_{2}\left(\mathrm{CH}_{3} \mathrm{COO}\right)_{4}\left(\mathrm{H}_{2} \mathrm{O}\right)_{2}$ & $\mathrm{Cu}_{2} \mathrm{O}_{7}$ \\
\hline CASCI & +78 & -253 & -33 & -452 \\
DDCI1 & -9 & -745 & -114 & -903 \\
DDCI2 & -21 & -815 & -120 & -952 \\
DDCI & -15 & -1125 & -238 & -1137 \\
$J_{\text {exp }}$ & $0,-40^{\mathrm{a}}$ & $<-800^{\mathrm{b}}$ & $-286^{\mathrm{c}},-294 \pm 4^{\mathrm{d}}$ & $-1032 \pm 48^{\mathrm{e}}$ \\
& & & & $-1081 \pm 40^{\mathrm{f}}$ \\
\hline
\end{tabular}

\begin{tabular}{ll}
\hline${ }^{\mathrm{a}}$ Reference 62. & ${ }^{\mathrm{d}}$ Reference 38. \\
${ }^{\mathrm{b}}$ Reference 63. & ${ }^{\mathrm{e}}$ Reference 40. \\
${ }^{\mathrm{c}}$ Reference 64. & ${ }^{\mathrm{f}}$ Reference 41.
\end{tabular}


TABLE VII. Contributions to the coupling constant, $J$ (in $\mathrm{cm}^{-1}$ ), using natural orbitals.

\begin{tabular}{lcccr}
\hline \hline & {$\left[\mathrm{Cu}_{2} \mathrm{Cl}_{6}\right]^{2-}$} & {$\left[\mathrm{Cu}_{2}\left(\mathrm{~N}_{3}\right)_{2}\left(\mathrm{NH}_{3}\right)_{6}\right]^{2+}$} & $\mathrm{Cu}_{2}\left(\mathrm{CH}_{3} \mathrm{COO}\right)_{4}\left(\mathrm{H}_{2} \mathrm{O}\right)_{2}$ & $\mathrm{Cu}_{2} \mathrm{O}_{7}$ \\
\hline Direct exchange & 162 & 720 & 66 & 334 \\
Kinetic exchange & -84 & -973 & -99 & -786 \\
$1 h+1 p$ & -87 & -492 & -81 & -451 \\
$2 h, 2 p$ & -12 & -70 & -6 & -49 \\
$2 h+1 p, 1 h+2 p$ & 6 & -310 & -118 & -185 \\
\hline Total & -15 & -1125 & $-286^{\mathrm{c}},-294 \pm 4^{\mathrm{d}}$ & -1137 \\
$J_{\text {exp }}$ & $0-40^{\mathrm{a}}$ & $<-800^{\mathrm{b}}$ & & $-1032 \pm 48^{\mathrm{e}}$ \\
& & & & -238 \\
\hline \hline
\end{tabular}

${ }^{\text {a Reference } 62 .}$

${ }^{\mathrm{b}}$ Reference 63 .

${ }^{c}$ Reference 64.

dedicated CI technique. However the result essentially consisted in a number, and any physical analysis of the factors leading to the final value of $J$ was lacking. This prevented any confrontation with the qualitative models, popular among the specialists of the domain, and frequently used as a tool in the design of new magnetic architectures. The present work is an attempt to overcome this difficulty and to show that it is possible from accurate calculations to analyze the physical effects contributing to the observable. While perturbative approaches provide a natural way for such analysis (and we actually have followed this way for analytic derivation and qualitative considerations), they are not quantitatively reliable, since many partial series are too slowly convergent (for instance the dynamical polarization of ionic VB structures). We have combined localization of the magnetic orbitals into atom centered magnetic orbitals and appropriate partitions of the CI space, in order to reach the desired information.

The most relevant conclusions are the following:

(1) The physics cannot be kept restricted to the valence space, with the simple balance between the direct $(K)$ and kinetic $\left(-4 t^{2} / U\right)$ exchanges, whatever the definition of the valence space (mean field variational or natural magnetic orbitals), the action of the exact Hamiltonian in this restricted space leads to values of $J$ which are one order of magnitude smaller than experiment (and sometimes even of incorrect sign).

(2) The spin polarization is non-negligible, although is not the main effect beyond the valence space. Its nature (ferro or antiferro) is system-dependent.

(3) The main effect beyond the CAS and belonging to the DDCI2 list, (built from second order arguments) comes from $1 h+1 p$ outer space determinants and is a fourth (and higher) order correction. It consists in the dynamical repolarization of the ionic VB structures.

(4) The processes involving two inactive holes or two inactive particles have a much smaller effect.

(5) Beyond the DDCI2 space, which is not sufficient to reach a quantitative agreement with experiment, one must involve $2 h+1 p$ and $1 h+2 p$ excitations. Their effect is large. It is not a dynamical polarization of the ligand to metal charge transfer states, but it proceeds through a dynamical coupling of the ligand-metal transi-
${ }^{\mathrm{d}}$ Reference 38.

${ }^{\mathrm{e}}$ Reference 40.

${ }^{\mathrm{f}}$ Reference 41. tions dipole with transition dipoles of the surrounding electrons, and an increase of the effective hopping integral of dispersive origin.

At this stage, it may be interesting to see whether and how these different physical effects are included in alternative $a b$ initio techniques. The NOCI method certainly does not include the spin polarization effects since it works with restricted open-shell SCF descriptions of each VB form. Since it only introduces a limited number of $l \rightarrow a$ charge transfer states (with their specific $h \rightarrow p$ relaxations), selected on rather intuitive considerations, it certainly lacks part of the $2 h+1 p$ and $1 h+2 p$ effects, which have opposite trends. The importance of the lacking contributions may depend on the nature of the bridging-ligands.

The CASPT2 approach, when starting from the valence CI space (minimal CAS), introduces the effects of all DDCI perturbers and in principle does not miss any of the above considered effects. However, it is a contracted scheme and does not revise the composition of the valence part of the wave function, namely the ionic/neutral ratio in the singlet state. As shown in paper II ${ }^{58}$ the dynamical correlation effects dramatically increase this ratio (multiplied by a factor between 2 and 5) through higher order effects, which are incorporated in a variational treatment. Using contracted schemes results in an underestimation of the perturbation, especially when polarizable ligands are present in the molecular structure. In these cases, the recipe consists in enlarging the CAS to include in this way part of the higher order effects. A detailed discussion comparing CASPT2 and DDCI methods will be given elsewhere.

This paper has performed the above analysis with two rather different sets of orbitals, the Hartree-Fock ones, and quasinatural MOs. The magnetic orbitals are significantly more delocalized on the ligands in the second set, as phenomenologically observed, ${ }^{57}$ and rationalized here. This effect leads to larger zero-order values of the direct exchange, $K$, and the hopping integral $t$. The DDCI2 values become more accurate when natural orbitals are used, but the $2 h$ $+1 p$ and $1 h+2 p$ excitations still have a non-negligible effect on the final value of $J$.

The present analysis of the physical effects that contribute to the magnetic coupling may seem quite complex. At 
this stage it seems to invalidate the models that essentially stay within the valence-only space. However the next paper ${ }^{58}$ shows that the application of the effective Hamiltonian theory, makes it possible to return to a simplified picture, i.e., to the valence model space. The use of effective energies and interactions modified in order to incorporate the complex and massive effects of the outer space excitations allows us to return closer to qualitative pictures.

\section{ACKNOWLEDGMENTS}

These two works have been stimulated by the lectures given (in particular by M. Verdaguer) in the ESF Tutorial on Theoretical Aspects of Molecular Magnetism, Vienna, November 2000. The authors are indebted to C. de Graaf for many comments and suggestions. They thank the SpanishFrench Scientific Cooperation (Integrated Action HF20000030). J.C. and R.C. want to thank the DGICYT of the Ministerio de Educación y Cultura of Spain (Project No. PB981216-CO2-02) and the CIRIT of the Generalitat de Catalunya (Grant No. SGR99-182) for their financial support. C.J.C. acknowledges the financial support through the TMR activity "Marie Curie research training grants" Grant No. HPMF-CT-1999-00285 of the European Commission. The Laboratoire de Physique Quantique is Unité Mixte de Recherche (UMR 5626) du CNRS.

${ }^{1}$ (a) M. Verdaguer, A. Bleuzen, V. Marvaud et al., Coord. Chem. Rev. 190-192, 1023 (1999); (b) M. Verdaguer, Polyhedron 20, 1115 (2001).

${ }^{2}$ Molecular Magnetism: From Molecular Assemblies to the Devices, NATO ASI Series. Series E: Applied Sciences, edited by E. Coronado, P. Delhaès, D. Gatteschi, and J. S. Miller (Kluwer, Dordrecht, 1995), Vol. 321. ${ }^{3}$ O. Kahn, Molecular Magnetism (VCH, New York, 1993).

${ }^{4}$ (a) W. Heisenberg, Z. Phys. 49, 619 (1928); P. A. M. Dirac, Proc. R. Soc. London, Ser. A 123, 714 (1929); (b) P. A. M. Dirac, The Principles of Quantum Mechanics (Clarendon, Oxford, 1947); (c) J. H. Van Vleck, The Theory of Electric and Magnetic Susceptibilities (Oxford University Press, Oxford, 1932).

${ }^{5}$ F. Fabrizi de Biani, E. Ruiz, J. Cano, J. J. Novoa, and S. Alvarez, Inorg. Chem. 39, 3221 (2000)

${ }^{6}$ E. Ruiz, J. Cano, S. Alvarez, and P. Alemany, J. Am. Chem. Soc. 120, 11122 (1998).

${ }^{7}$ C. Adamo, V. Barone, A. Bencini, F. Totti, and I. Ciofini, Inorg. Chem. 38, 1996 (1999).

${ }^{8}$ (a) R. L. Martin and F. Illas, Phys. Rev. Lett. 79, 1539 (1997); (b) F. Illas and R. L. Martin, J. Chem. Phys. 108, 2519 (1998).

${ }^{9}$ (a) A. Bencini, D. Gatteschi, and C. Zanchini, Inorg. Chem. 24, 704 (1985); (b) A. Bencini and D. Gatteschi, J. Am. Chem. Soc. 108, 5763 (1986).

${ }^{10}$ J. Caro, P. Alemany, S. Alvarez, M. Verdaguer, and E. Ruiz, Chem.-Eur. J. 4, 476 (1998).

${ }^{11}$ (a) L. Noodleman and J. G. Norman, Jr., J. Chem. Phys. 70, 4903 (1979); (b) L. Noodleman, ibid. 74, 5737 (1981); (c) L. Noodleman and E. R. Davidson, Chem. Phys. 109, 131 (1986); (d) L. Noodleman, C. Y. Peng, D. A. Case, and J. M. Mouesca, Coord. Chem. Rev. 144, 199 (1995).

${ }^{12}$ R. Caballol, O. Castell, F. Illas, I. de P. R. Moreira, and J. P. Malrieu, J. Phys. Chem. A 101, 7860 (1998).

${ }^{13}$ R. Broer and W. C. Nieuwpoort, Theor. Chim. Acta 73, 405 (1998).

${ }^{14}$ (a) A. B. van Oosten, R. Broer, and W. C. Nieuwpoort, Chem. Phys. Lett. 257, 207 (1996); (b) Int. J. Quantum Chem., Quantum Chem. Symp. 29, 241 (1995)

${ }^{15}$ A. B. van Oosten and F. Mila, Chem. Phys. Lett. 295, 359 (1998).

${ }^{16}$ K. Andersson, Theor. Chim. Acta 73, 405 (1988).

${ }^{17}$ (a) K. Andersson, P.-Å. Malmqvist, B. O. Roos, A. J. Sadlej, and K. Wolinski, J. Phys. Chem. 94, 5483 (1990); (b) K. Andersson, P.-A. Malmqvist, and B. O. Roos, J. Chem. Phys. 96, 1218 (1992).

${ }^{18}$ C. de Graaf, R. Broer, and W. C. Nieuwpoort, Chem. Phys. Lett. 271, 372 (1997).
${ }^{19}$ S. Yamanaka, M. Okumura, H. Nagao, and K. Yamaguchi, Chem. Phys. Lett. 233, 88 (1995).

${ }^{20}$ (a) C. de Graaf, I. de P. R. Moreira, and F. Illas, Int. J. Mol. Sci. 1, 28 (2000); (b) C. Sousa, C. de Graaf, F. Illas, and G. Pacchioni, Prog. Theor. Phys. 7, 227 (2000); (c) C. de Graaf, C. Sousa, I. de P. R. Moreira, and F. Illas, J. Phys. Chem. A 105, 11371 (2001).

${ }^{21}$ A. Ceulemans, G. A. Heylen, L. F. Chiboatru, T. L. Maes, K. Pierloot, C. Ribbing, and L. G. Vanquickenborne, Inorg. Chim. Acta 251, 15 (1996).

${ }^{22}$ J. Miralles, O. Castell, R. Caballol, and J. P. Malrieu, Chem. Phys. 172, 33 (1993).

${ }^{23}$ J. Miralles, J. P. Daudey, and R. Caballol, Chem. Phys. Lett. 198, 555 (1992).

${ }^{24}$ O. Castell, R. Caballol, V. M. García, and K. Handrick, Inorg. Chem. 35, 1609 (1996)

${ }^{25}$ O. Castell and R. Caballol, Inorg. Chem. 38, 668 (1999).

${ }^{26}$ J. Cabrero, N. Ben Amor, C. de Graaf, F. Illas, and R. Caballol, J. Phys. Chem. A 104, 9983 (2000).

${ }^{27}$ I. de P. R. Moreira, F. Illas, C. J. Calzado, J. F. Sanz, J. P. Malrieu, N. Ben Amor, and D. Maynau, Phys. Rev. B 59, R6593 (1999).

${ }^{28}$ (a) C. J. Calzado, Ph.D. thesis, University of Sevilla, Spain, 1998, (b) C. J. Calzado, J. F. Sanz, J. P. Malrieu, and F. Illas, Chem. Phys. Lett. 307, 102 (1999); (c) C. J. Calzado, J. F. Sanz, and J. P. Malrieu, J. Chem. Phys. 112, $5158(2000)$

${ }^{29}$ N. Suaud and M. B. Lepetit, Phys. Rev. B 62, 402 (2000).

${ }^{30}$ (a) C. J. Calzado and J. P. Malrieu, Eur. Phys. J. B 21, 375 (2001); (b) C. J. Calzado and J. P. Malrieu, Phys. Rev. B 63, 214520 (2001).

${ }^{31}$ P. de Loth, P. Cassoux, J. P. Daudey, and J. P. Malrieu, J. Am. Chem. Soc. 103, 4007 (1981).

${ }^{32}$ R. Broer and W. J. A. Maaskant, Chem. Phys. 102, 103 (1986).

${ }^{33}$ O. Castell, J. Miralles, and R. Caballol, Chem. Phys. 179, 377 (1994).

${ }^{34}$ O. Kahn, in Magneto-Structural Correlation in Exchange Coupled Systems, NATO Advanced Studies Series. C, edited by R. D. Willett, D. Gatteschi, and O. Khan (Reidel, Dordrecht, 1985), Vol. 140, p. 389.

${ }^{35}$ M. F. Charlot, O. Kahn, M. Chaillet, and C. Larrieu, J. Am. Chem. Soc. 108, 2574 (1986).

${ }^{36}$ J. W. Steed, B. J. McCool, and P. C. Junck, J. Chem. Soc. Dalton Trans. 1998, 3417.

${ }^{37}$ D. N. Hendrickson, in Magneto-Structural Correlation in Exchange Coupled Systems, NATO Advanced Studies Series. C, edited by R. D. Willett, D. Gatteschi, and O. Khan (Reidel, Dordrecht, 1985), Vol. 140, p. 525.

${ }^{38}$ H. U. Güdel, A. Stebler, and A. Furer, Inorg. Chem. 18, 1021 (1979).

${ }^{39}$ A. H. Ewald and E. Sinn, Inorg. Chem. 8, 537 (1969).

${ }^{40}$ (a) P. E. Sulewski, P. A. Fleury, K. B. Lyons, S. W. Cheong, and Z. Fisk, Phys. Rev. B 41, 225 (1990); (b) R. P. Singh, P. A. Fleury, K. B. Lyons, and P. C. Sulewski, Phys. Rev. Lett. 62, 2736 (1989).

${ }^{41}$ (a) G. Aeppli, S. M. Hayden, H. A. Mook, Z. Fisk, S. W. Cheong, D. Rytz, J. P. Remeika, G. P. Espinosa, and A. S. Cooper, Phys. Rev. Lett. 62, 2052 (1989); (b) Y. Endoh, K. Yamada, R. J. Birgeneau et al., Phys. Rev. B 37, 7443 (1988); (c) S. M. Hayden, G. Aeppli, R. Osborn, A. D. Taylon, T. G. Perring, S. W. Cheong, and Z. Fisk, Phys. Rev. Lett. 67, 3622 (1991)

${ }^{42}$ (a) W. E. Pickett, Rev. Mod. Phys. 61, 433 (1989); (b) Y.-S. Su, T. A. Kaplan, and S. D. Mahanti, Phys. Rev. B 59, 10521 (1999).

${ }^{43}$ Z. Barandiarán and L. Seijo, Can. J. Chem. 70, 409 (1992).

${ }^{44}$ K. Pierloot, B. Dumez, P.-O. Widmark, and B. O. Roos, Theor. Chim. Acta 90, 87 (1995)

${ }^{45}$ S. Huzinaga, L. Seijo, Z. Barandiarán, and M. Klobukowski, J. Chem. Phys. 86, 2132 (1987)

${ }^{46}$ W. J. Hehre, R. F. Stewart, and J. A. Pople, J. Chem. Phys. 51, 2657 (1969).

${ }^{47}$ P. J. Hay and W. R. Wadt, J. Chem. Phys. 82, 299 (1985).

${ }^{48}$ (a) T. H. Dunning, J. Chem. Phys. 53, 2823 (1970); (b) T. H. Dunning and P. J. Hay, in Methods of Electronic Structure Theory, edited by H. F. Schaefer III (Plenum, New York, 1977), Vol. 2.

${ }^{49}$ MOLCAS version 4, K. Andèrsson, M. R. A. Blomberg, M. P. Fülscher et al., Lund University, Sweden, 1997.

${ }^{50}$ CASDI program, N. Ben Amor and D. Maynau, Chem. Phys. Lett. 286, 211 (1998)

${ }^{51}$ NATURAL program, V. M. García, O. Castell, and R. Caballol, Rovirai Virgili University, Tarragona, Spain (1997).

${ }^{52}$ (a) P. W. Anderson, Phys. Rev. 79, 350 (1950); (b) P. W. Anderson, in Theory of the Magnetic Interaction: Exchange in Insulators and Superconductors, edited by F. Turnbull and F. Seitz (Academic Press, New York, 1963), Vol. 14, p. 99. 
${ }^{53}$ O. Kahn and B. Briat, J. Chem. Soc., Faraday Trans. 2 72, 268 (1976).

${ }^{54}$ P. J. Hay, J. C. Thibeault, and R. Hoffmann, J. Am. Chem. Soc. 97, 488 (1975).

${ }^{55}$ W. Geertsma, Ph.D. thesis, University of Groningen, The Netherlands, 1989; Physica B 164, 241 (1990).

${ }^{56}$ H. Eskes and H. Jefferson, Phys. Rev. B 48, 9788 (1993).

${ }^{57}$ J. Cabrero, C. J. Calzado, D. Maynau, R. Caballol, and J. P. Malrieu (submitted).

${ }^{58}$ C. J. Calzado, J. Cabrero, J. P. Malrieu, and R. Caballol, J. Chem. Phys. (in press).

${ }^{59}$ F. Illas, I. de P. R. Moreira, C. de Graaf, O. Castell, and J. Casanovas, Phys. Rev. B 56, 5069 (1997).
${ }^{60}$ C. de Graaf, I. de P. R. Moreira, F. Illas, and R. L. Martin, Phys. Rev. B 60, 3457 (1999)

${ }^{61}$ D. Muñoz, F. Illas, and I. de P. R. Moreira, Phys. Rev. Lett. 84, 1579 (2000).

${ }^{62}$ (a) R. D. Willett, in Magneto-Structural Correlation in Exchange Coupled Systems, NATO Advanced Studies Series. C, edited by R. D. Willett, D. Gatteschi, and O. Khan (Reidel, Dordrecht, 1985), Vol. 140, p. 389; (b) G. Maass, B. Gerstein, and R. D. Willett, J. Chem. Phys. 46, 401 (1967); (c) G. O'Bannon and R. D. Willett, Inorg. Chim. Acta 53, 6131 (1983).

${ }^{63}$ P. Chaudhuri, K. Oder, K. Wieghardt, B. Nuber, and J. Weiss, Inorg. Chem. 25, 2818 (1986).

${ }^{64}$ B. N. Figgis and R. L. Martin, J. Chem. Soc. 1956, 3837. 